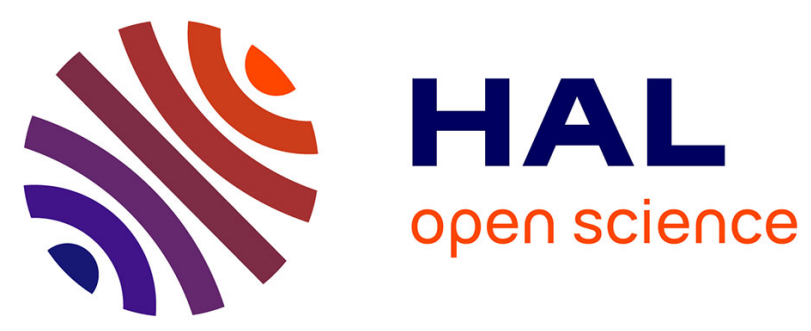

\title{
Histamine releasing factor and elongation factor 1 alpha secreted via malaria parasites extracellular vesicles promote immune evasion by inhibiting specific $\mathrm{T}$ cell responses
}

Claudia Demarta-Gatsi, Anna Rivkin, Vincenzo Di Bartolo, Roger Peronet, Shuai Ding, Pierre-Henri Commère, Francois Guillonneau, Jacques Bellalou, Sébastien Brûlé, Paula Abou Karam, et al.

\section{- To cite this version:}

Claudia Demarta-Gatsi, Anna Rivkin, Vincenzo Di Bartolo, Roger Peronet, Shuai Ding, et al.. Histamine releasing factor and elongation factor 1 alpha secreted via malaria parasites extracellular vesicles promote immune evasion by inhibiting specific T cell responses. Cellular Microbiology, 2019, 21 (7), 10.1111/cmi.13021 . hal-02571995v2

\section{HAL Id: hal-02571995 \\ https://univ-angers.hal.science/hal-02571995v2}

Submitted on 29 May 2020

HAL is a multi-disciplinary open access archive for the deposit and dissemination of scientific research documents, whether they are published or not. The documents may come from teaching and research institutions in France or abroad, or from public or private research centers.
L'archive ouverte pluridisciplinaire HAL, est destinée au dépôt et à la diffusion de documents scientifiques de niveau recherche, publiés ou non, émanant des établissements d'enseignement et de recherche français ou étrangers, des laboratoires publics ou privés. 


\title{
Histamine releasing factor and elongation factor 1 alpha secreted via malaria parasites extracellular vesicles promote immune evasion by inhibiting specific $\mathrm{T}$ cell responses
}

\author{
Claudia Demarta-Gatsi ${ }^{1,2,3}$ | Anna Rivkin ${ }^{4}$ | Vincenzo Di Bartolo ${ }^{5}$ | Roger Peronet ${ }^{1,2,3}$ | \\ Shuai Ding ${ }^{1,2,3}$ | Pierre-Henri Commere ${ }^{6}$ | François Guillonneau ${ }^{7}$ | Jacques Bellalou ${ }^{8}$ | \\ Sébastien Brûlé ${ }^{9}$ | Paula Abou Karam ${ }^{4}$ | Sidney R. Cohen ${ }^{10}$ | Thibault Lagache ${ }^{11}$ | \\ Chris J. Janse ${ }^{12}$ | Neta Regev-Rudzki ${ }^{4}$ | Salaheddine Mécheri ${ }^{1,2,3}$ (D)
}

${ }^{1}$ Institut Pasteur, Unité de Biologie des Interactions Hôte Parasites, Paris, France

${ }^{2}$ Department of Parasites and Insect vectors, Institut Pasteur, CNRS ERL9195, Paris, France

${ }^{3}$ Department of Parasites and Insect vectors, Institut Pasteur, INSERM U1201, Paris, France

${ }^{4}$ Department of Biomolecular Sciences, Weizmann Institute of Science, Rehovot, Israel

${ }^{5}$ Institut Pasteur, Lymphocyte Cell Biology Unit, Department of Immunology, INSERM U1221, Paris, France

${ }^{6}$ Institut Pasteur, Imagopole, Paris, France

${ }^{7} 3 \mathrm{P} 5$ proteomics Facility of the Université Paris Descartes, Institut Cochin, Paris, France

${ }^{8}$ Platform of Recombinant Proteins, C2RTInstitut Pasteur, Paris, France

${ }^{9}$ Platform of Molecular Biophysics, Institut Pasteur, Paris, France

${ }^{10}$ Department of Chemical Research Support, Weizmann Institute of Science, Rehovot, Israel

${ }^{11}$ Department of Biological Sciences, Columbia University, New York, New York

${ }^{12}$ Leiden Malaria Research Group, Parasitology, Leiden University Medical Center (LUMC), Leiden, The Netherlands

\section{Correspondence}

Salaheddine Mécheri, Institut Pasteur, Unité de Biologie des Interactions Hôte Parasites, Paris, France.

Email: smecheri@pasteur.fr

Funding information

Israel Science Foundation (ISF), Grant/Award Numbers: 619/16 and119034, 119034 and 619/ 16; European Research Council (ERC), Grant/ Award Number: 757743; Helmut Horten Foundation, Agno, Switzerland; Institut Pasteur; Pasteur-Weizmann joint research program; French Parasitology consortium ParaFrap, Grant/ Award Number: ANR-11-LABX0024

\section{Abstract}

Protozoan pathogens secrete nanosized particles called extracellular vesicles (EVs) to facilitate their survival and chronic infection. Here, we show the inhibition by Plasmodium berghei NK65 blood stage-derived EVs of the proliferative response of $\mathrm{CD} 4^{+} \mathrm{T}$ cells in response to antigen presentation. Importantly, these results were confirmed in vivo by the capacity of EVs to diminish the ovalbumin-specific delayed type hypersensitivity response. We identified two proteins associated with $\mathrm{EVs}$, the histamine releasing factor (HRF) and the elongation factor 1a (EF-1a) that were found to have immunosuppressive activities. Interestingly, in contrast to WT parasites, EVs from genetically HRF- and EF-1a-deficient parasites failed to inhibit $T$ cell responses in vitro and in vivo. At the level of T cells, we demonstrated that EVs from WT parasites dephosphorylate key molecules (PLC $\gamma 1$, Akt, and ERK) of the T cell receptor signalling cascade. Remarkably, immunisation with EF-1a alone or in combination with HRF conferred a long-lasting antiparasite protection and immune memory. In conclusion, we identified a new mechanism by which P. berghei-derived EVs exert their immunosuppressive functions by altering $T$ cell responses. The identification of two highly conserved immune suppressive factors offers new conceptual strategies to overcome EV-mediated immune suppression in malaria-infected individuals.

\section{KEYWORDS}

extracellular vesicles, immunosuppression, immune evasion, malaria, T cells 


\section{1 | INTRODUCTION}

Protozoan pathogens use a number of different strategies to avoid host immune destruction. In malaria parasites, antigenic variation has been shown to contribute to chronic infection (Scherf, Lopez-Rubio, \& Riviere, 2008). However, this mechanism alone cannot account for the prolonged chronic infections in human malaria patients. Recent findings demonstrate that extracellular vesicles (EVs) play an important role in many biological processes including pathogen survival and propagation (Feng et al., 2013; Ramakrishnaiah et al., 2013). In the human malaria parasite Plasmodium falciparum, EVs have been first described as a mode of cell-cell communication (Mantel et al., 2013; Regev-Rudzki et al., 2013) during the asexual blood stage. Studies carried out in malaria experimental models have shown that Plasmodium iRBC-derived EVs induce macrophage activation in a TLR-dependent fashion in vitro (Couper et al., 2010). In support of these observations, P. falciparum iRBCs-derived EVs have been shown to stimulate the cells of the innate immune system in vitro by activating a proinflammatory and anti-inflammatory cytokine response in macrophages and by strongly activating neutrophils to spontaneously move even in the absence of a chemotactic gradient (Mantel et al., 2013). Recently, Regev-Rudzki and colleagues revealed a previously unidentified aspect: $P$. falciparum strains deliver genomic DNA via EVs to alter the immune response (Sisquella et al., 2017).

EVs are nanosized particles, ranging from $50 \mathrm{~nm}$ to $1 \mu \mathrm{m}$, limited by a lipid bilayer that contains proteins, nucleic acids, and lipids derived from the parent cell. Depending on their size, biogenesis, and cellular compartment of origin, three EV subtypes can be distinguished. Several distinct types of EVs have been reported in the literature (Malda, Boere, van de Lest, van Weeren, \& Wauben, 2016; Zhang et al., 2018), among them, apoptotic bodies together with microvesicles and exosomes with sizes ranging between 1,0005,000 nm, 100-1000 nm, and 50-200 nm, respectively, comprise the most prominent groups of EVs (van Dongen, Masoumi, Witwer, \& Pegtel, 2016). Exosomes are spherical vesicles with a bilamellar membrane and specific density formed within endosomes by invagination of the limiting membrane, resulting in the formation of multivesicular bodies (Raposo \& Stoorvogel, 2013; Thery, 2011). In contrast, microvesicles are formed by budding and shedding from the plasma membrane and are larger with greater size heterogeneity. EVs carry diverse content such as RNA (Baglio et al., 2016; Ridder et al., 2015), DNA (Sansone et al., 2017; Sisquella et al., 2017; Thakur et al., 2014), proteins (Simpson, Lim, Moritz, \& Mathivanan, 2009; Torrecilhas, Schumacher, Alves, \& Colli, 2012), and lipids (Record, Silvente-Poirot, Poirot, \& Wakelam, 2018; Verderio, Gabrielli, \& Giussani, 2018), which can be delivered to target cells.

Previously, we had reported that histamine releasing factor (HRF) exerts a strong immunomodulating activity in the experimental malaria model Plasmodium berghei and that the deletion of this gene leads to a long-lasting protective immune response in mice (Demarta-Gatsi et al., 2016; Demarta-Gatsi et al., 2017). Here, we show that HRF exerts its immunosuppressive activities via its association to EVs. HRF in combination with another EVs factor called elongation factor $1 a$ (EF-1a) inhibits antigen-specific $T$ cell responses through interference with key phosphorylation pathways associated with T cell receptor (TCR) signalling. Our results show that immunosuppressive EVs are part of the strategy employed by Plasmodium parasites to escape the host immune response. Furthermore, this work will impact malaria vaccine development research aiming long-lasting antiparasite immune responses.

\section{2 | RESULTS}

\section{1 | Morphological characterisation of iRBCs derived EVs}

We have established a protocol for EV production and purification from cultured $P$. berghei infected $\mathrm{RBC}(\mathrm{Pb} \mathrm{iRBC})$ supernatants using a combination of ultracentrifugation/filtration procedures. Dynamic light scattering (DLS), a technique that allows determination of the particle size distribution and possibly protein contamination, was used as quality control of the purification procedure. In addition, other techniques such as tunable resistive pulse sensing (TRPS), nanoparticle tracking analysis (NTA), and atomic force microscopy (AFM) were used to provide additional features of the isolated EVs (Figure S1A-D, respectively). AFM measurements identified rounded EVs with sizes within the range found by the other techniques (Figure S1D). There was no detectable variation in the shape of EVs from different sources. By combining data obtained from DLS, TRPS, NTA, and AFM analysis, we can conclude that our vesicles have size and circular structure corresponding to those of exosomes. As we cannot formally preclude the possibility of other existing vesicles, we therefore termed them "extracellular vesicles (EVs)."

\subsection{Analysis of the protein content of iRBCs derived EVs}

On the basis of the previous studies performed in Leishmania (Nandan, Yi, Lopez, Lai, \& Reiner, 2002) and Trypanosoma (Opperdoes \& Szikora, 2006; Silverman \& Reiner, 2011), we hypothesised that Plasmodium parasites may secrete virulence effectors associated to EVs that may interfere with host immune responses. Liquid chromatographytandem mass spectrometry (LC-MS/MS) of the collected EVs from $\mathrm{Pb}$ iRBCs was performed to identify, as comprehensively as possible, the proteins secreted into EVs that may be involved in virulence. Proteomic analysis was performed in five independent experiments, and only proteins that were reproducibly found in all experiments, the most confidently represented, and that display at least two peptides were considered for analysis. In total, 151 proteins were identified originating from parasites, after excluding database redundancy (Table S1). This included 16 proteins (Table S2) that confirm the exosomal nature of the preparation, because they are often found in exosomes and classified in the top 100 exosomal protein markers in ExoCarta (http://exocarta.org/exosome_markers_new), a web-based collection of exosomes that displays a list of exosomal components such as proteins, mRNA, and miRNA. From this, we selected a list of 
proteins that were found to be the mostly represented in Plasmodium derived EVs (Table S3). Remarkably, several parasite antigens were found to be associated with EVs including merozoite surface proteins (MSP) 1, EF-1a, and heat-shock proteins. These results are compatible with previous studies carried out in Plasmodium (Abdi et al., 2017; Martin-Jaular, Nakayasu, Ferrer, Almeida, \& Del Portillo, 2011) and Leishmania (Opperdoes \& Szikora, 2006; Silverman et al., 2010) parasites where these proteins were detected in exosome-like vesicles released during the infection. Altogether, these results show that EVs obtained from $\mathrm{Pb}$ iRBC cultures were derived from the infected cells. The presence of MSP1, EF-1a, and HSP70 in Plasmodium EVs was confirmed by western blotting using anti-MSP1-33, anti-EF-1a, or anti-HSP70 antibodies (Figure S1A-C, respectively), showing bands at the expected size of 28,55 , and $70 \mathrm{kDa}$, respectively.

Additionally, western blot studies on purified EVs (Figure S2D) revealed the presence of HRF in $P$. berghei parasites using an antibody specific to that protein. Previous studies showed that HRF is exported via a nonclassical pathway involving exosomal exit (Amzallag et al., 2004) in 293T and HepG2 cell lines. Moreover, the protein was not detected in PbNK65-hrf $\Delta$ iRBCs or in uninfected RBCs (uRBCs)derived EVs, further confirming the presence of HRF in PbNK65 EVs.

\section{3 | P. berghei iRBC-derived EVs inhibit concanavalin A-induced cell proliferation}

We set out to understand whether and how EVs derived from $P$. berghei parasites may contribute to malaria pathogenesis by exerting immunomodulatory activities on innate and adaptive immune responses. As a model system, we first assessed a splenic $\mathrm{T}$ cell response to a mitogen knowing the importance of the spleen in the elimination of parasites and in the development of the immune response during malaria infection both in humans and in experimental murine models (Bachmann et al., 2009; Bereczky et al., 2006; Greenwood et al., 1987; Looareesuwan et al., 1987; Oomen, Meuwissen, \& Gemert, 1979). Spleen cells obtained from C57BL/6J mice were labelled with carboxyfluorescein succinimidyl ester (CFSE), a fluorescent dye used to monitor leukocyte proliferation, and incubated for $24 \mathrm{hr}$ with $1 \mathrm{\mu g} / \mathrm{ml}$ of concanavalin A (ConA) in the presence of different concentrations of purified EVs (Figure 1). According to this procedure, when CFSE-labelled cells divide, their progeny only retained half of the number of CFSE-tagged molecules, and thus, each cell division can be assessed by measuring the corresponding decrease in cell fluorescence. Spleen cells were stained with anti-CD3, and the effect of parasite EVs on T cell proliferation was monitored by flow cytometry. As expected, unstimulated cells (Figure 1a, CTL panel) and EVs added to cells in the absence of ConA (Figure 1a, EV $1 \mu \mathrm{g} / \mathrm{ml}$ panel) did not show any sign of proliferation, whereas the ConA control cells (Figure 1a, ConA panel) showed cell division cycles, with $46.27 \%$ of the cells proliferating in response to ConA. In contrast, T cell proliferation was completely inhibited in the presence of PbNK65 iRBC EVs at concentrations of 3 and $1 \mu \mathrm{g} / \mathrm{ml}$. For a lower concentration of $0.1 \mu \mathrm{g} / \mathrm{ml}$, no inhibition occurred. A quantitative analysis is shown in Figure $1 \mathrm{~b}$ where the proportion of dividing (M2) and nondividing cells (M1) stimulated with ConA in the presence of various doses of EVs is represented. These results clearly show an inhibitory effect of parasite EVs on a mitogen-induced $T$ cell proliferation.

\section{4 | P. berghei iRBC-derived EVs inhibit antigen-specific $T$ cell proliferation}

Activation of $T$ cells requires stimulation through the TCR by a specific antigen presented by the appropriate major histocompatibility (a)

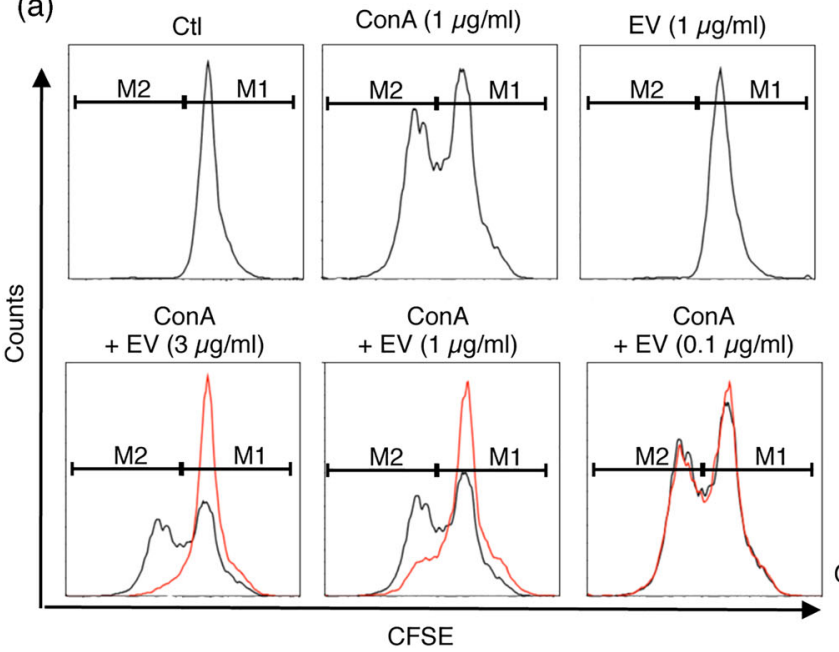

(b)

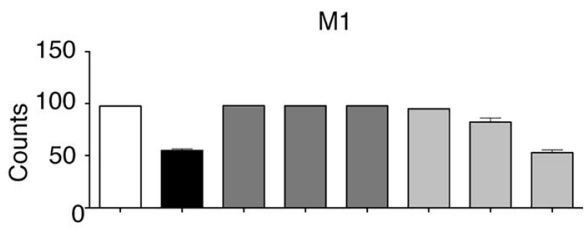

$\mathrm{M} 2$

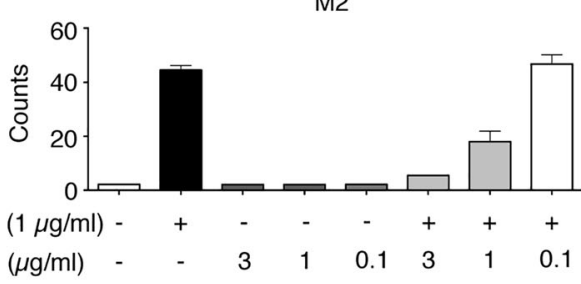

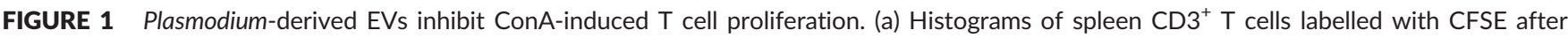
$24 \mathrm{hr}$ culture in the presence of $1 \mu \mathrm{g} / \mathrm{ml}$ of ConA and the indicated PbNK65 iRBCs-derived EV concentrations (lower panels). The M1 window corresponds to resting cells, whereas the M2 window contains proliferating cells. Upper panels show the different controls. (b) Quantitative representation of non-proliferating (M1) and proliferating (M2) $C D 3^{+} \mathrm{T}$ cells in the presence of ConA. Error bars, SEM. Data are representative of three independent experiments with three mice per group 
molecule (MHC) on antigen-presenting cells (APCs). We first considered the possibility that EVs containing parasite antigens can affect the dendritic cell (DC) function in its capacity to process the antigen into peptides, loading onto $\mathrm{MHC}$ molecules, and present at the plasma membrane to activate effector $T$ cells. Bone marrow-derived DCs were preincubated with $100 \mu \mathrm{g} / \mathrm{ml}$ ovalbumin (OVA) in the presence of different concentrations of purified PbNK65 iRBCs EVs or noninfected RBCs EVs derived from naïve mice for $24 \mathrm{hr}$, then washed and incubated with CFSE-stained OVA-specific CD4 ${ }^{+} \mathrm{T}$ cells from the lymph nodes of OT-II mice (Figure 2a). Cell division was assessed after $48 \mathrm{hr}$ incubation by measuring CFSE dilution by flow cytometry, as explained above (Figure 2). Under these conditions, there was no impact on the $T$ cell proliferation because no inhibition was observed regardless of the presence or absence of EVs (Figure 2a, middle (a)

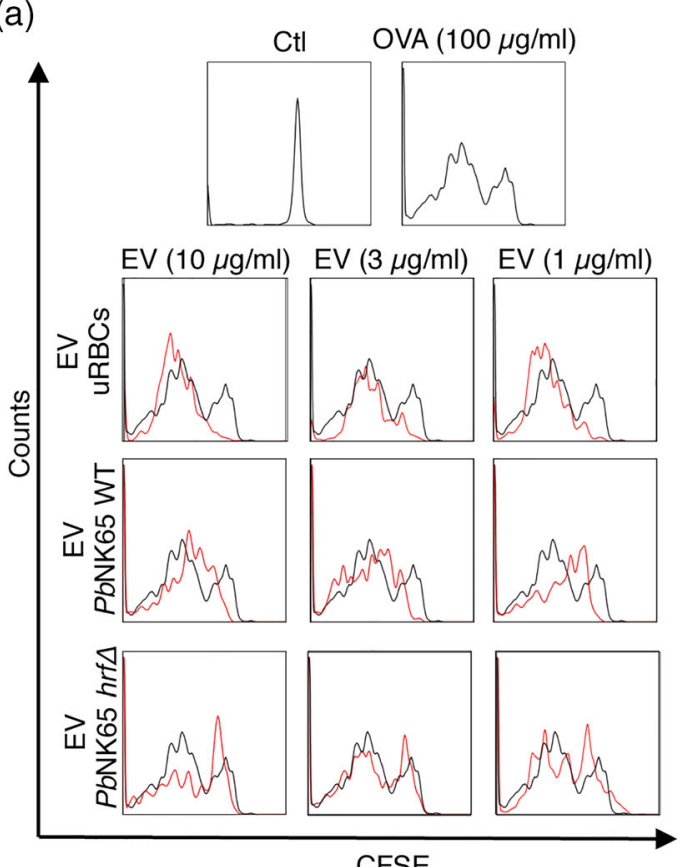

(c)

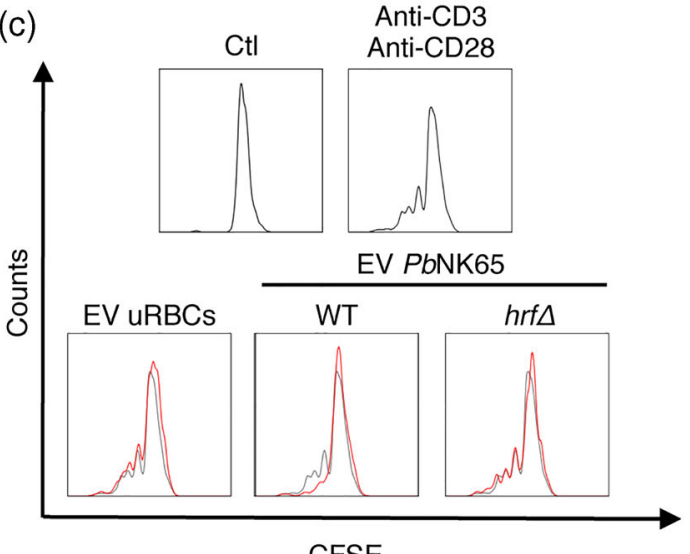

(b)

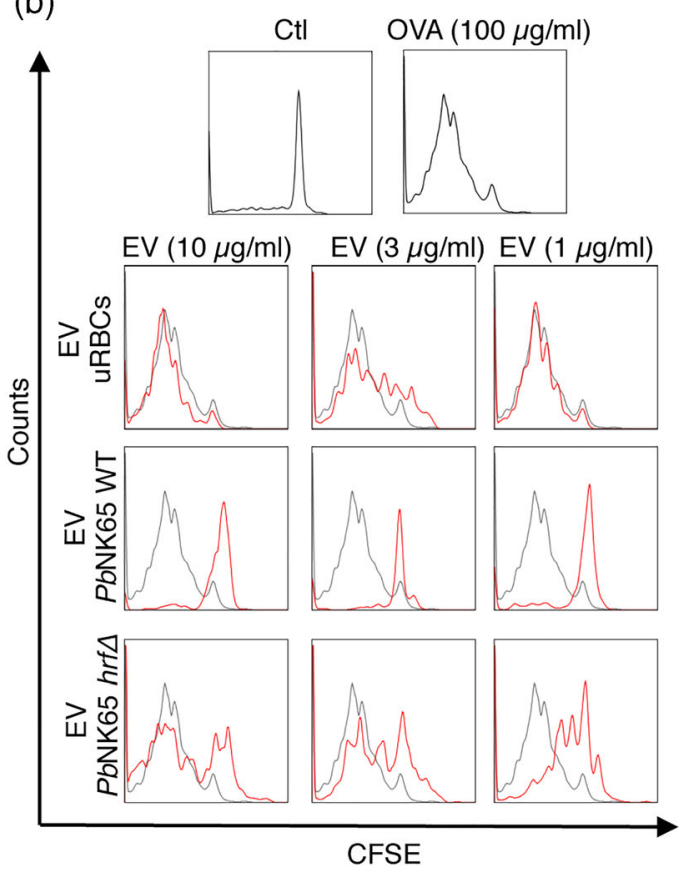

(d)
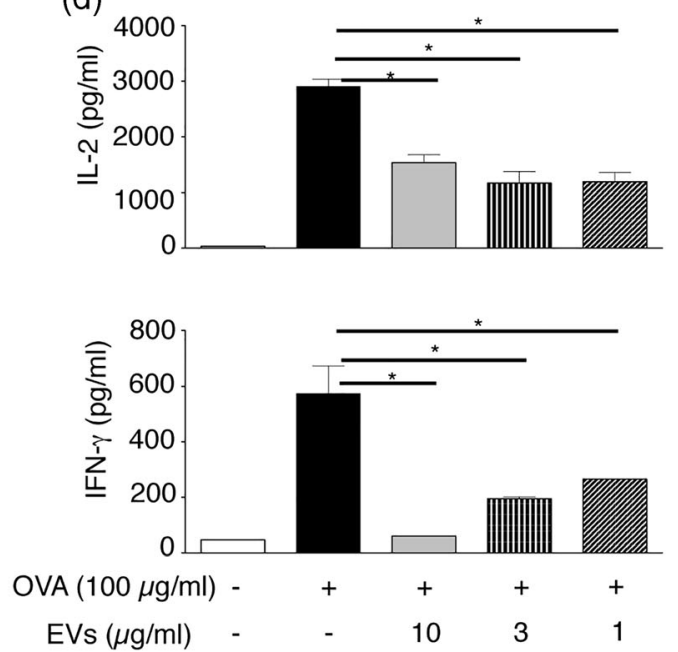

FIGURE 2 Inhibition by Plasmodium-derived EVs of antigen-specific T cell proliferation and cytokine release in vitro. CFSE-labelled OT-II CD4 ${ }^{+} \mathrm{T}$ cells were (a) cultured with DCs which were first incubated with OVA and EVs from different sources and washed prior to addition to OT-II T cells or (b) cocultured altogether with DCs, OVA, and EVs from different sources. After $48 \mathrm{hr}$ incubation, proliferation was measured by flow cytometry as dilution of CFSE dye. (c) CFSE-labelled T cells from lymph nodes of C57BL/6J mice were cultured in the presence of anti-CD3/CD28 Dynabeads, $3 \mu \mathrm{g} / \mathrm{ml} \mathrm{EVs}$, and $30 \mathrm{U} / \mathrm{ml} \mathrm{IL-2}$. After 6 days of culture, CFSE fluorescence was assessed by flow cytometry. Upper panels show negative and positive proliferation controls. Lower panels show T cell proliferation in the presence of EVs from different sources (red histograms) and in the absence of EVs (black histograms). Data are representative of six independent experiments with two replicates per group. (d) Levels of IL-2 and IFN- $\gamma$ cytokines production by OVA-specific CD4 ${ }^{+} T$ cells from the lymph nodes of OT-II mice and bone marrow-derived DCs cultures incubated for $24 \mathrm{hr}$ with different concentrations of purified EVs were measured in the culture supernatants by ELISA. Error bars, SEM. Data are representative of six independent experiments with two replicates per group. ${ }^{*} P<0.05$; Mann-Whitney test 
row). This preincubation of EVs with DCs did not result in any change in any surface activation markers such as MHC II, CD80, and CD86, suggesting that EVs had apparently no effect on the antigen presenting function of DCs (data not shown). Alternatively, a bulk culture of DCs and $\mathrm{CD}^{+} \mathrm{T}$ cells from the lymph nodes of OT-II mice were cultured altogether in the presence of $100 \mu \mathrm{g} / \mathrm{ml} \mathrm{OVA}$ and EVs (Figure 2b). In these conditions, the proliferation of OT-II-stimulated T cells was completely inhibited at all doses used when PbNK65 WT iRBC-EVs were added to the cocultures (Figure $2 \mathrm{~b}$, middle row) as compared with OVA alone or to EVs from uRBCs (Figure $2 b$, upper row). This inhibition of $T$ cell proliferation was functionally concomitant with EV-mediated inhibition of IL-2 and IFN- $\gamma$ secreted by T cells (Figure 2d). Additionally, on the basis of our previous observations that the parasite HRF was directly associated with the disease pathogenesis, and given its association with EVs, we decided to compare the inhibitory effects of PbNK65 WT and hrf $\triangle$ iRBC-EVs on T cell proliferation/activation. As shown in Figure $2 \mathrm{~b}$, bottom row, PbNK65 hrf $\triangle \mathrm{iRBC}$-EVs were much less efficient in inhibiting $T$ cell proliferation than the highly effective WT EVs (Figure 2b, middle row). These results demonstrate that EVs from WT parasites are involved in antigen-specific $T$ cell inhibition and that this inhibition is in part mediated by HRF suggesting that during Plasmodium infection, this mechanism may reflect how parasite-derived EVs contribute to the development of the disease. Data shown in Figure 2a,b strongly suggest, but do not formally demonstrate, that EVs more likely act on T cells rather than on the APC functions. To mimic the antigen presentation assay and to demonstrate that EVs indeed exert their T cell inhibitory effects independently of APCs, we adopted a T cell proliferation assay where $T$ cells were activated directly by crosslinking the TCR using a cocktail of anti-CD3- and anti-CD28-coated beads. In this study, we examined the effect of an optimal dose of $3 \mu \mathrm{g} / \mathrm{ml}$ EVs. As shown in Figure 2c, similar results were obtained in that PbNK65 WT had inhibitory and hrf $\mathrm{iRBC}$ EVs had noninhibitory effects on $\mathrm{T}$ cell proliferation

These findings prompted us to characterise the uptake of $\mathrm{Pb}$ iRBCs EVs by CD4 and CD8 T cells. Thiazole orange (TO)-labelled EVs were cultured with lymph node cells and incubated for various periods of time. T cells were labelled with anti-CD4 and anti-CD8 antibodies and analysed by imaging flow cytometry on the ImageStreamX, as previously reported (Ofir-Birin et al., 2018; Figure 3). EVs were rapidly taken up by both CD4 (Figure 3a) and CD8 (Figure 3b) T cells within 1 min incubation, and this uptake as assessed by fluorescenceactivated cell sorter (FACS) analysis was time dependent, because the percentage of double positive cells, $\mathrm{TO}^{+} \mathrm{CD}^{+}$and $\mathrm{TO}^{+} \mathrm{CD}^{+}$, increased with time from $1 \mathrm{~min}$ to $1 \mathrm{hr}$ (Figure 3c). To preclude any toxic effect of EVs on T cells, and to ascertain that the inhibition of the observed $\mathrm{T}$ cell proliferation was not due to possible cytotoxic effects, CFSE-labelled T cells were stained with 7-amino actinomycin

(b)

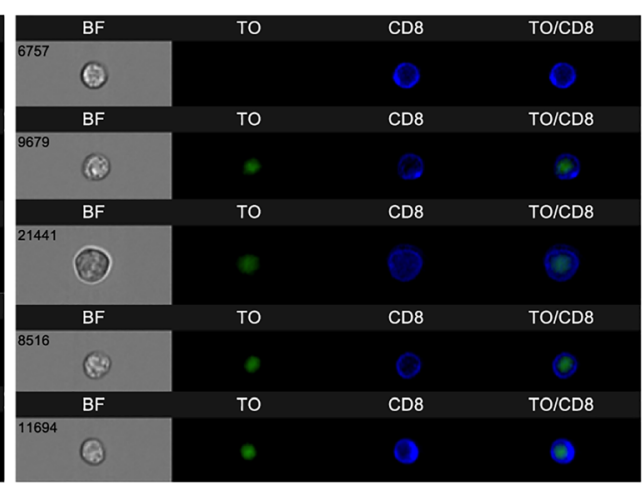

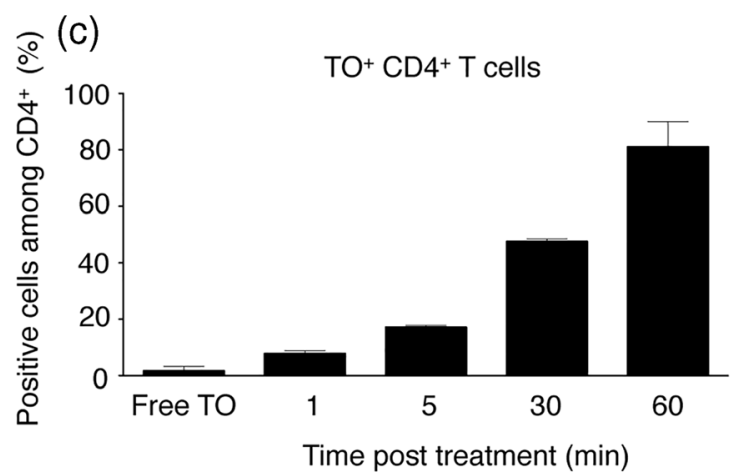

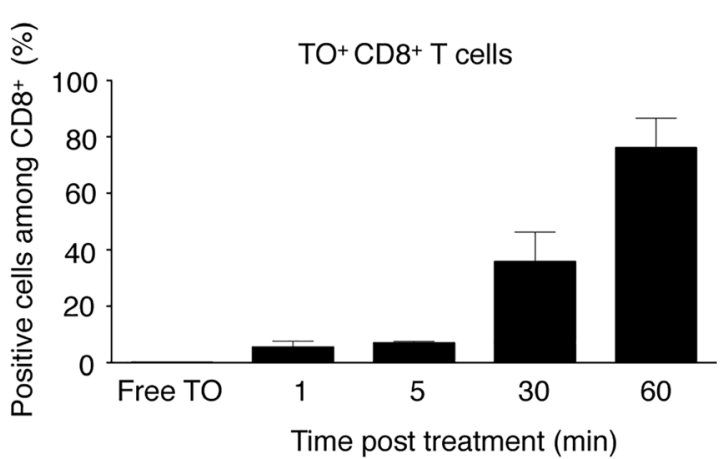

FIGURE 3 Uptake of Plasmodium berghei-derived EVs by T cells. (a, b) Total lymph node cells were incubated for different periods of time (1 min, $5 \mathrm{~min}, 30 \mathrm{~min}$, and $1 \mathrm{hr}$ ) with Thiazole orange (TO)-labelled EVs (this dye specifically labels RNA). CD4 ${ }^{+}$and CD8 ${ }^{+} \mathrm{T}$ cells labelled with Alexa Fluor 700 and Pacific blue, respectively, were imaged by imaging flow cytometry (IFC). (c) Percentage of TO-labelled positive CD4 ${ }^{+}$and CD ${ }^{+} \mathrm{T}_{\text {cells }}$ gated according to unlabelled EVs. Controls consisted of cells incubated with the same dilution of TO (free TO) that stained EVs. Error bars, SEM. Data are representative of three independent experiments with two mice per group 
D, a nonpermeant dye used to identify nonviable cells, and were monitored by flow cytometry. As shown in Figure S3, EVs at all doses used did not show any toxic effect on $\mathrm{T}$ cells.

\section{5 | Attenuation by P. berghei iRBC-derived EVs of antigen-specific delayed type hypersensitivity response in vivo}

In order to confirm the results obtained in vitro, we performed a delayed type hypersensitivity (DTH) analysis where cell-mediated immune responses associated with Th1 reactivity were evaluated in vivo. The DTH reaction is divided into the afferent and efferent phases. During the afferent phase mice are immunised by subcutaneous injection with OVA emulsified in complete Freund's adjuvant at the base of the tail. One week later, $30 \mu \mathrm{l}$ of aggregated OVA with $10 \mu \mathrm{g} \mathrm{EV} /$ mouse were injected in one hind footpad and the same volume of phosphate buffered saline (PBS) in the other. At $24 \mathrm{hr}$ postchallenge, the DTH response, initiated by antigen-specific $T$ cells that had previously proliferated and differentiated into effector $\mathrm{CD} 4^{+}$IFN $-\gamma^{+}$T cells, was evaluated by measuring the swelling of the footpad. Measurement of the footpad thickness showed a diminished swelling by $P b N K 65$ WT EVs whereas inhibition was not seen with either URBC-derived EVs or with PbNK65 hrf $\mathrm{EV}$ s (Figure 4a). In parallel, cell counts of total leukocytes in draining lymph nodes were measured, and the net increase showed no significant difference (Figure 4 b). Consistent with the DTH response, percentage and total $\mathrm{CD}^{+}$ IFN $-\gamma^{+}$cells were significantly reduced by PbNK65 WT EVs but not with control PbNK65 uRBCs (Figure 4c,d). Taken together, these data demonstrate that EVs have an inhibitory effect on $\mathrm{T}$ cell function in vivo and that this inhibition is mediated by the HRF protein associated with EVs.

To verify whether EVs mediate their inhibitory effects independently of the mutated gene or if this is a unique feature of the hrf

\section{EVs}

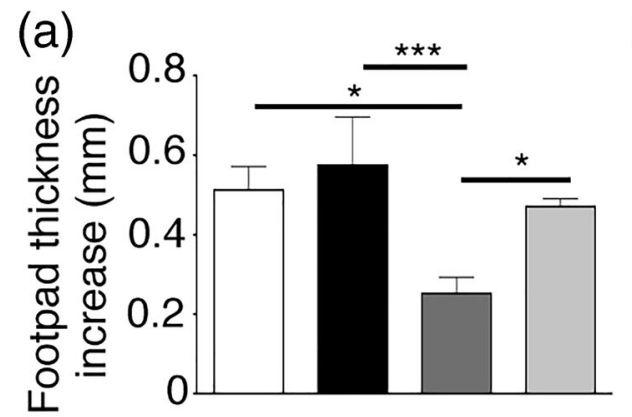

(b)

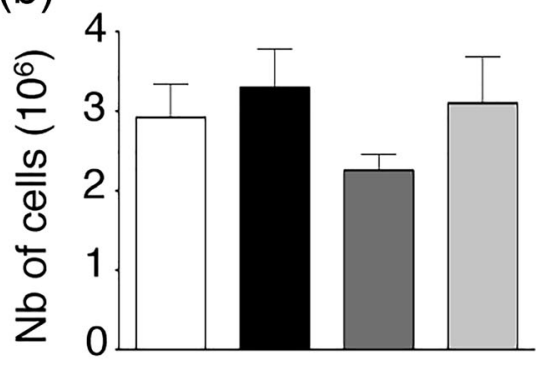

\section{$\mathrm{CD} 4+$ IFN- $\gamma^{+}$}

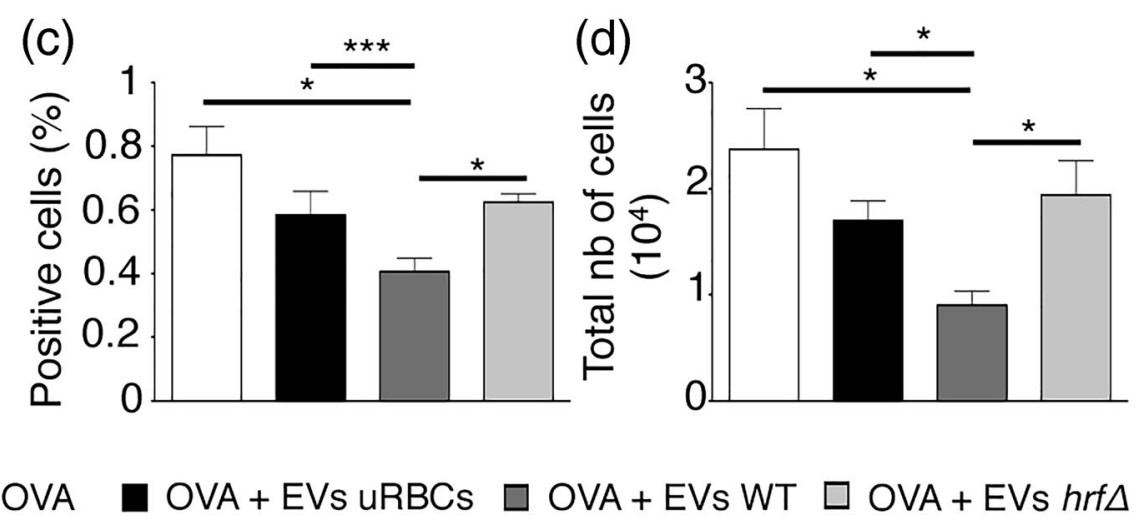

FIGURE 4 EVs from WT parasites but not from mutant parasites attenuate the delayed type hypersensitivity (DTH) response. The DTH reaction was performed by immunising mice with OVA in complete Freund's adjuvant. Seven days later, mice were challenged with a s.c. injection in the right footpad of emulsified OVA combined with parasite EVs or with PBS in the left footpad as control. (a) Swelling was measured after 24 hr using a calliper rule. Results are displayed as mean difference \pm standard deviation in footpad thickness of left versus right footpad. (b) Counts of total popliteal lymph node leukocytes. Representative frequency (c) and absolute number (d) of CD4 ${ }^{+} \mathrm{T}$ cells harvested after a 24 hr DTH reaction, cultured for $4 \mathrm{hr}$ with PMA + ionomycine and followed by intracellular staining of IFN- $\gamma$ as analysed by flow cytometry. Error bars, SEM. Data are representative of three independent experiments with five mice per group; ${ }^{*} P<0.0095,{ }^{* *} 0.0167<P<0.0242,{ }^{* * *} 0.0317<P<0.0424$, MannWhitney test 
gene, we prepared EVs from various Plasmodium parasites including two mutants for PbANKA EF-1a genes (Janse et al., 2003; line 115, $e E F-1 a b \Delta$ and line 118, eEF-1aa) that indeed showed no protein products as assessed by western blotting (Figure S2b) and a PbANKA mutant where the gene encoding for the high mobility group 2 (HMGB2) protein was deleted (PbANKA $h m g b 2 \Delta$ ), and the disruption of this gene was found previously to attenuate $\mathrm{PbANKA}$ pathogenicity (Briquet et al., 2015). As shown in Figure S4A, similar to $h r f \Delta$ parasites, EVs from the two lines, line $115 \mathrm{eEF}-1 \mathrm{ab}$ and line $118 \Delta e E F-1 a a$, were no longer able to inhibit the DTH response. By contrast, hmgb2 $\Delta$ parasites preserved their ability to inhibit the DTH response in a manner similar to WT parasites. In addition, the measurements of CD4 $4^{+} \mathrm{IFN}-\gamma^{+}$ T cells, a characteristic feature of the DTH response, showed an inhibition of the percentage and the total number of cells upon injection of $h m g b 2 \Delta$ EVs similar to WT EVs, whereas EVs from hrf $\Delta$ parasites had no inhibitory effects (Figure S4B,C).

The gating strategy for FACS analysis is shown in Figure S4D. These data strongly suggest that the in vivo inhibitory effects of $\mathrm{Pb}$ EVs on the antigen-specific $T$ cell response does not seem to be the panacea of any genetically deficient parasite but rather HRF and EF$1 a$ are only particular gene products that are endowed with this biological activity. We questioned whether EVs from mutant parasites could not inhibit $\mathrm{T}$ cell functions just because of a lack of uptake by these T cells. This was proven not to be the case because these EVs were taken up with the same efficacy as EVs from WT parasites (data not shown). This may suggest that these two genes either alter common signalling pathways in T cells or act in coordination and in a concerted manner to inhibit T cell functions. Lastly, we evaluated whether recombinant $P b$ proteins would similarly affect the DTH and CD4 T cell response. As shown in Figure S4E-H, both recombinant PbHRF and PbEF-1a inhibit the DTH response and the induction of $\mathrm{CD}^{+}$ IFN $-\gamma^{+} \mathrm{T}$ cells. It must be pointed out that mixing the two proteins did not display any synergistic effect.

\subsection{HRF and EF-1a colocalise in P. berghei parasites and interact with each other}

Infected RBCs with GFP-labelled parasites were examined by confocal microscopy, and the distribution of HRF and the EF-1a in $\mathrm{Pb}$ parasites was assessed by labelling with AlexaFluor 568 and AlexaFluor 647, respectively. Both proteins were uniformly distributed in the parasite, with areas of coincident brighter staining (Figure 5a). Except for a pattern of EF-1a molecules that shows a localisation at the RBC surface, the HRF and the EF-1 $a$ detected within the parasite were clustered and colocalised (Figure 5a). For statistical analysis, we designed a multistep approach using Icy software in which we first defined manually a region of interest (ROI) around the parasite GFP signal (Figure 5b) and measured overlap between segmented HRF and EF-1a masks with Manders coefficients M1 and M2 in the Icy plugin Colocalization Studio (Lagache, Sauvonnet, Danglot, \& Olivo-Marin, 2015). Overlap analysis indicated an important colocalisation of the two molecules (Figure $5 \mathrm{c}$ ). These data were consistent with biochemical approaches where pull-down experiments using either crude extracts of parasites (Figure $5 \mathrm{~d}$, left) or mixtures of recombinant HRF and EF-1a proteins (Figure 5D, right) eluted from anti-HRF- or anti-EF-1a-coated beads showed that the two proteins were physically associated. As shown in Figure 5 e, antibody-coated beads bind specifically to their respective proteins because no heterologous proteins could be eluted from either beads, which indicates the true existence of complexes between HRF and EF-1a molecules.

\section{7 | EV-mediated inhibition of $\mathrm{T}$ cell activation affects the phosphorylation of key TCR-associated proteins}

Inhibition of antigen-specific $T$ cell responses in vitro and in vivo prompted us to examine biochemical pathways that might be affected by $\mathrm{Pb}$ EVs. The engagement of TCR and CD28 induces the phosphorylation of multiple key proteins including PLC $\gamma 1$, Akt, and ERK. Hence, lymph node $T$ cells isolated from naïve mice were subjected to TCR and CD28 costimulation for various periods of time in the absence or in the presence of EVs from PbNK65 WT, or PbNK65 hrf $\Delta$, or PbANKA ef1 $a-a \Delta$, or PbANKA ef $1 a-b \Delta$ parasites. EVs were used at the dose of $3 \mu \mathrm{g} / \mathrm{ml}$ that was found to be optimal based on in vivo (DTH response) and in vitro (antigen presentation) assays. Cells were subsequently lysed and analysed by immunoblotting with antiPhospho PLC $\gamma 1$, pAKT, and pERK antibodies. As shown in Figure 6, in the absence of EVs (CTL), the phosphorylation increased sharply after 2 min of activation and progressively decreased after 5 min and returned to the basal level after $10 \mathrm{~min}$. When EVs from PbNK65 WT parasites were added to activated T cells, a significant decrease in the intensity of phosphorylated proteins was observed at 2 min after activation. In contrast to WT EVs, PbNK65 hrf $\triangle \mathrm{EVs}$ had significantly less inhibitory effect on the phosphorylation of the same proteins (Figure 6a). To provide a quantitative estimate, band intensities for each phosphoprotein were normalised to the GAPDH relative amount in the same lane (Figure 6b). Similarly, in contrast to EVs from WT parasites, those obtained from PbNK65 hrf $\Delta$ or PbANKA ef1a- $a \Delta$ failed to inhibit the phosphorylation of the same proteins (Figure 6c,d), except that of ERK 1/2. These data indicate that EVs down modulate antigen-specific $T$ cell responses by interfering with key phosphorylation events associated with the TCR engagement and that the inhibition of $\mathrm{T}$ cell activation is associated with the presence of HRF and EF-1a.

\section{8 | Immunisation with P. berghei iRBC-derived EVs protect mice against infection}

Given the potent immunosuppressive activity of EVs on T cell activation, we postulated that immunising mice against these vesicles would protect them from infection. Outbred Swiss Webster (SW) mice and inbred C57BL/6 mice were repeatedly injected with whole EVs isolated from iRBCs, and after reaching high and stable levels of specific antibodies, mice were challenged with infecting doses of sporozoites 
(a)

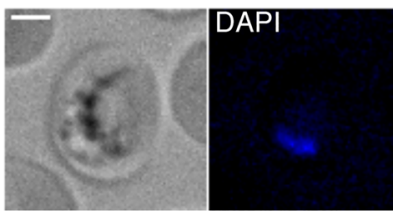

(b)

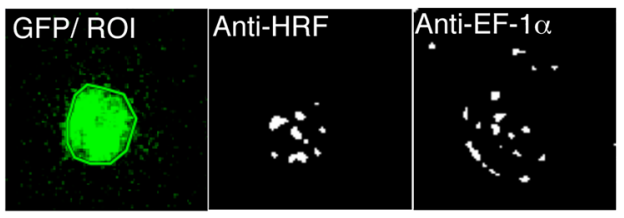

(d)

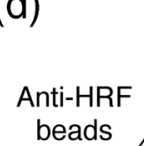

beads
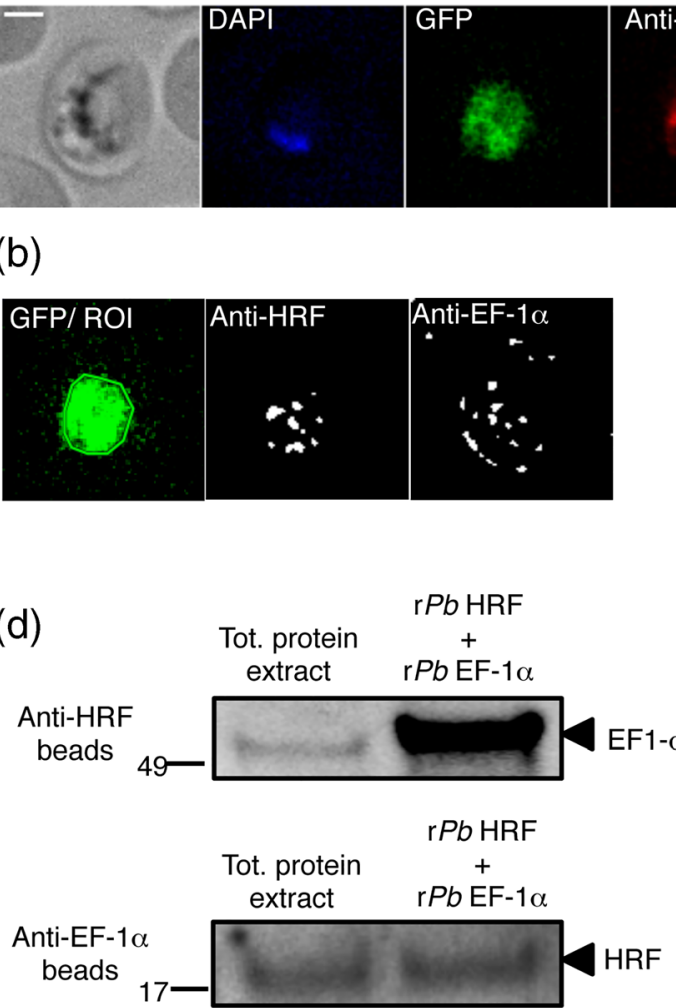
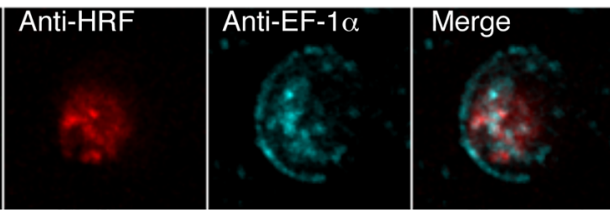

(c)

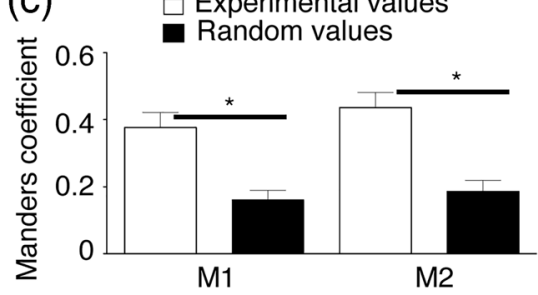

(e)

$\mathrm{rPb} \mathrm{HRF}$

$$
+
$$

$\mathrm{r} P b \mathrm{EF}-1 \alpha \quad \mathrm{r} P b \mathrm{EF}-1 \alpha$
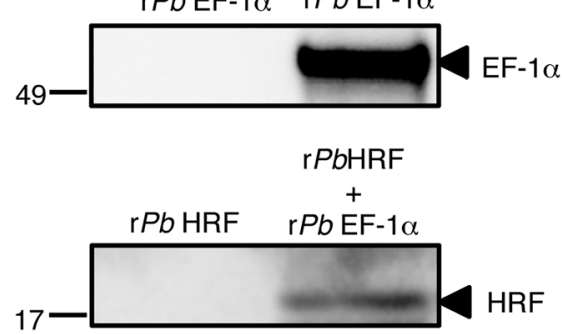

FIGURE 5 HRF and EF-1-a molecules colocalise within the parasite and interact with each other. (a) Infected RBCs with GFP-labelled parasites were stained with Alexa Fluor 558-anti HRF and with Alexa Fluor 647- anti EF-1a antibodies. Nuclei were stained with DAPI. The merge panel shows the colocalisation of the two proteins. Bar, $2 \mu \mathrm{m}$. (b) A region of interest (ROI) around GFP signal in each iRBC was manually drawn, and HRF and EF-1a clusters were segmented automatically using Icy software. (c) The overlap between segmented HRF and EF-1a masks with Manders coefficients in Icy plugin Colocalization Studio was measured (experimental values), and the statistical significance of measured M1 and M2 coefficients was calculated (random values). Finally, the empirical $P$ value with the rank order of experimental M1 and M2 compared with simulations was given $\left({ }^{*} P=0.012\right)$. (d) Pull-down experiments using anti-HRF or anti-EF-1a-coated beads on either total parasite protein extract or a mixture of $\mathrm{rPbHRF}$ and $\mathrm{rPbEF}-1 \mathrm{a}$ proteins followed by immunoblotting using alternate specific antibody. (e) Same experiment as in (d) except that the specificity of the pull-down experiments was tested

(SPZs) and parasitemia, and survival was monitored over time. As shown in Figure 7a,b, mice immunised with whole EVs and infected with SPZ-controlled parasitemia in the range of about 2 log and 1 log differences in SW mice and in C57BL/6 mice, respectively (highlighted in the inserts in the figure) as compared with control nonimmunised mice. From Day 15 postimmunisation (SW mice) and beyond (C57BL/6 mice), $80 \%$ and $20 \%$ of SW and C57BL/6 immunised mice, respectively, eliminated the parasites and survived the infection (Figure 7a,b). Considering EF-1a and HRF as potentially efficient vaccine candidates among EVs constituents, inbred C57BL/6 mice were immunised with recombinant PbEF-1a, and after mice developed high and stable amounts of anti- EF-1a antibodies, they were infected with either blood stage or SPZs. As shown in Figure $7 c$, mice immunised against $r P b E F-1 a$ were highly resistant to infection with SPZs because parasitemia was kept at a very low level until Day 30 postinfection; after which, parasites were completely cleared and $60 \%$ of mice survived the infection. Attempts to protect mice against infection following immunisation with recombinant $\mathrm{PbHRF}$ protein were not conclusive because the protein turned out to be poorly immunogenic in mice due to strong sequence homology between Plasmodium and mouse HRF (Figure 7d). Nevertheless, mice immunised with a mixture of $r P b E F-1 a$ and $r P b H R F$ acquired a strong protection against SPZ challenge because parasite clearance occurred 22 days postinfection, and all mice survived (Figure 7e). Control mice immunised with EVs from noninfected RBCs, which developed even higher parasitemia as compared with nonimmunised mice, were not able to control parasitemia and all died at Day 22 postinfection (Figure 7f). To determine whether surviving mice developed an immune memory against the parasites, all mice that survived the infection in each experimental group were challenged with 10,000 of PbNK65 SPZs. As shown in Figure S5, all immunised mice against EVs, rPbEF-1a, or against rPbEF-1a mixed with rPbHRF (Figure S5A$C$, respectively) and that cleared the parasite were completely free of parasite, indicating that they acquired a long-lasting antiparasite immune memory. These data demonstrate that immunisation with whole EVs or with the rPbEF-1a protein induce stage-transcendent long-lasting immune protection against Plasmodium parasite infections. 
(a)

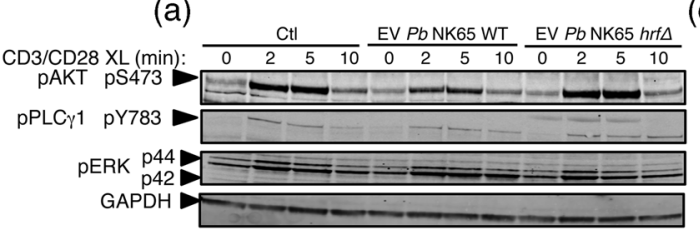

(b)

b) - Ctl - EV PbNK65 WT 七 EV PbNK65 hrtA

(c)
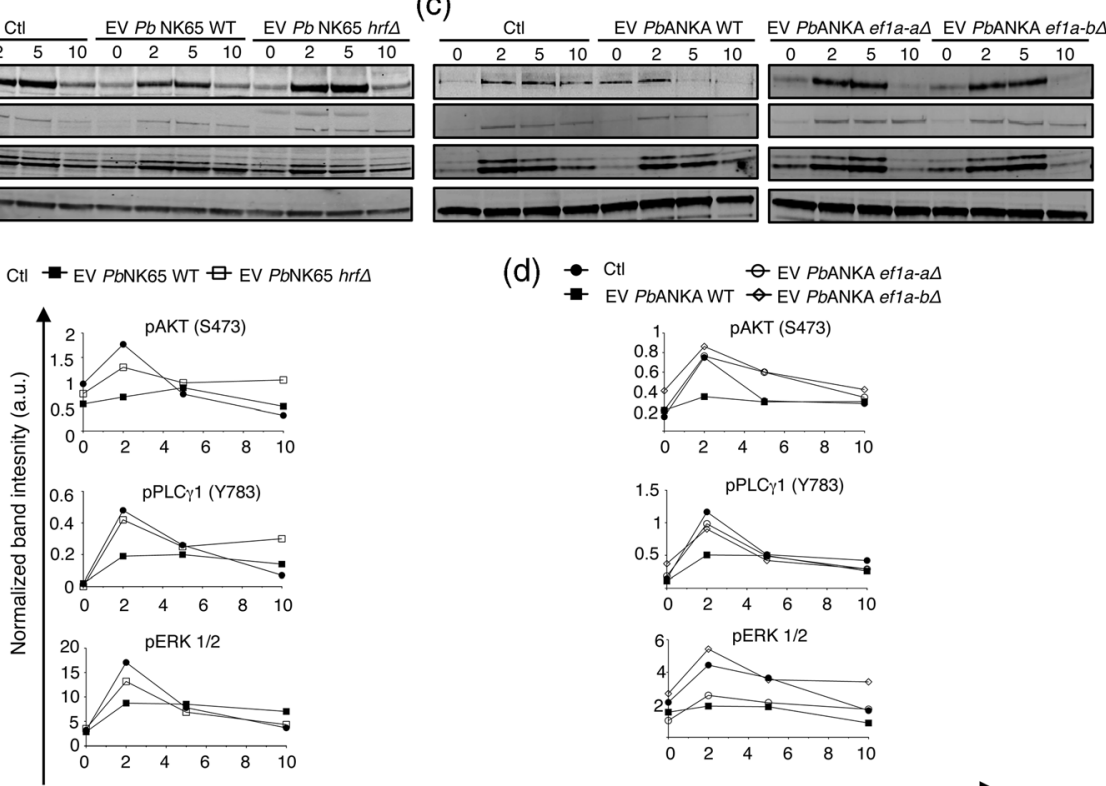

(d)

- EV PbANKA WT $\leadsto$ EV PbANKA ef1a-aA

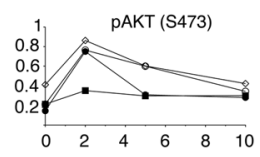

$\begin{array}{llll}6 & 8 & 10\end{array}$

$1.5 \quad$ PPLCy1 (Y783)

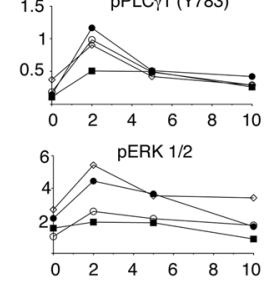

Time of stimulation (min)

FIGURE 6 Plasmodium-derived EVs reduce the phosphorylation level of proteins associated with the TCR signalling cascade. Lymph node cells from C57BL/6J mice were stimulated with anti-CD3 plus anti-CD28 antibody-coated beads in the absence or presence of parasite-derived EVs for the indicated time points. Cells were subsequently lysed and analysed by immunoblotting using antibodies against pAkt, $p$ PLC $\gamma 1$, and $p E R K 1 / 2$. Quantification of protein phosphorylation in the presence of EVs from PbNK65 WT, or PbNK65 hrf $\triangle$ (a, b) or EVs from PbANKA WT, or PbANKA ef $1 a-a \Delta$, or PbANKA ef $1 a-b \Delta$ parasites (c, d). Each band intensity was quantified and normalised to the GAPDH relative amount in the same lane. Normalised intensities, expressed as arbitrary units (a.u.) were then divided by the mean normalised intensity in the same experiment. In panel a, one single gel was used in which all samples could fit in. In panel b, running two separate gels was necessary, given the large number of samples. However, this has no incidence on data presentation and expression because internal controls were present for each sample. Data obtained from one single experiment replicated three to four times

\section{3 | DISCUSSION}

EV secretion is now considered as being an integral strategy of pathogens to promote their survival within their host (Schorey, Cheng, Singh, \& Smith, 2015). They transfer pathogen material of various origin such as protein, DNA, and RNA from the iRBC to host cells and have been shown to modulate immune responses (Ofir-Birin et al., 2018; Schorey \& Harding, 2016; Szempruch, Dennison, Kieft, Harrington, \& Hajduk, 2016). To our knowledge, this is the first report that describes a $T$ cell immunosuppressive function of EVs derived from Plasmodium parasites. Furthermore, we identify two molecular components of parasite EVs that act together to establish this T cell-oriented immunosuppression pathway. Deletion of the genes coding for these EVs components (HRF/EF-1) reverses the immunosuppression phenotype of EVs and links this observation directly to in vivo data that show a role of immune evasion for HRF in P. berghei-infected mice (Demarta-Gatsi et al., 2016; Demarta-Gatsi et al., 2017).

To identify the mechanism(s) by which PbEVs exert their inhibitory effects on T cells, proteomic and western blot analyses enabled us to identify two proteins among the PbEV cargo, HRF and EF-1a, which merited further scrutiny. The HRF protein, also known as translationally controlled tumour protein, P23/21, Q23, or fortilin, is a housekeeping protein, highly conserved in many organisms including several Plasmodium parasite species, and has no classical signal sequence comprising hydrophobic amino acids at its $\mathrm{N}$-terminal, which places it among the nontypically secreted proteins that exit from a cell without passing through the classical secretion pathway (Muesch et al., 1990). Indeed, it has been shown that HRF secretion proceeded via an endoplasmic reticulum/Golgi-independent or nonclassical pathway, associated to exosomes (Amzallag et al., 2004). Previous reports from our group have demonstrated that HRF, expressed by all Plasmodium parasites, when deleted in PbANKA or in PbNK65 parasites, caused a loss of virulence and induced a long-lasting sterile protection in P. berghei-infected mice (Demarta-Gatsi et al., 2016; Demarta-Gatsi et al., 2017). This supports the notion that HRF-containing EVs may represent a mechanism by which Plasmodium parasites disseminate their virulence and immunomodulatory factors to systemically reach out to various tissues including primary and secondary lymphoid organs where the antigen-specific $T$ cell response takes places. In this regard, inhibition of the T cell responses by HRF-containing EVs during antigen presentation to the immune system is of paramount significance. In support of the inhibitory effects of EVs observed in in vitro antigen presentation assays, $\mathrm{PbEVs}$ were also found to attenuate in vivo antigen-specific DTH responses, a typical IFN- $\gamma$-producing $\mathrm{CD}^{+} \mathrm{T}$ cell-mediated response, as reflected by the reduced footpad swelling upon antigen challenge. More importantly, HRF-deficient EVs produced by $P b H R F \Delta$-infected RBCs lost their capacity to inhibit the DTH response, suggesting that HRF represents a key virulence factor whose "packaging" into EVs may promote its long range activity by enhancing its stability and efficacy. 

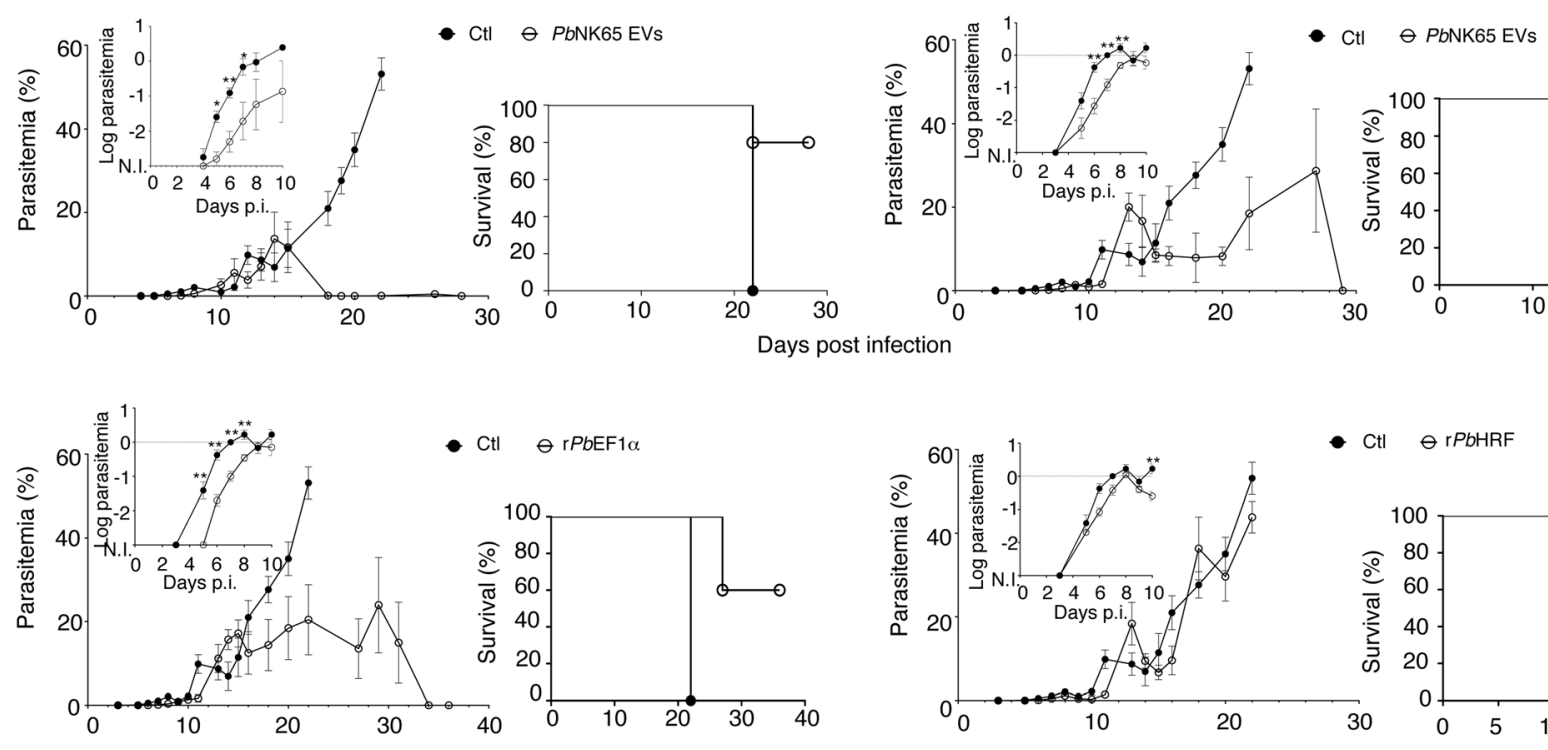

- Ctl $\theta \mathrm{rPbEF} 1 \alpha$

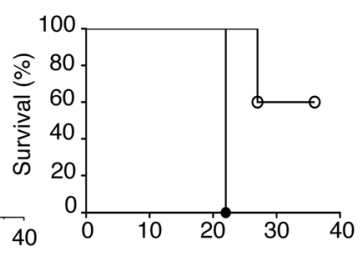

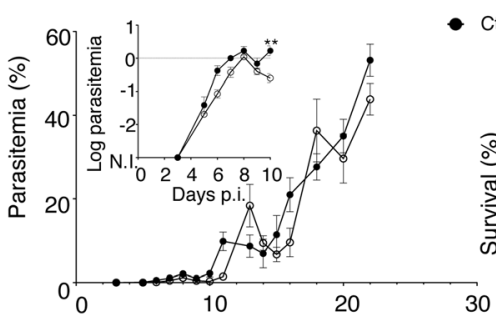

- Ctl $\theta \mathrm{rPbHRF}$

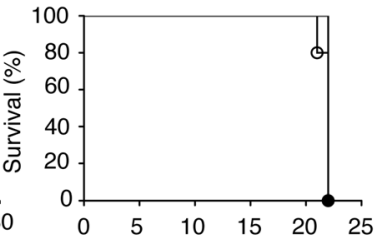

Days post infection

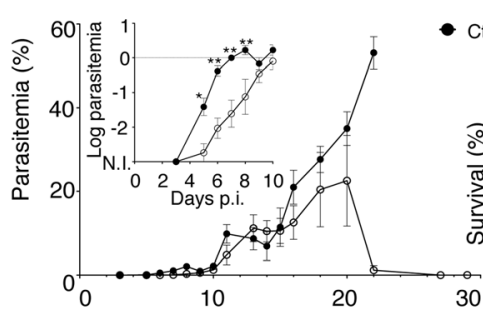

Ctl $\ominus$ rPbEF1 $\alpha+r P b H R F$

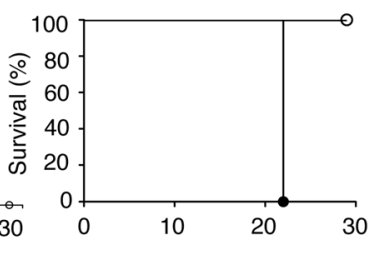

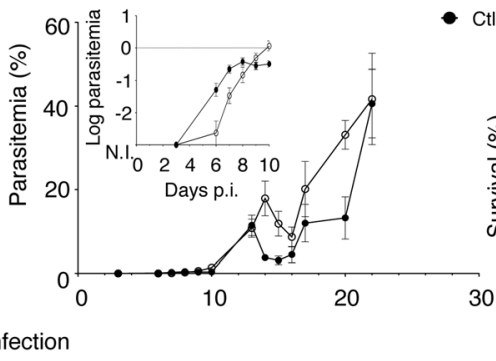

- $\mathrm{Ctl}$

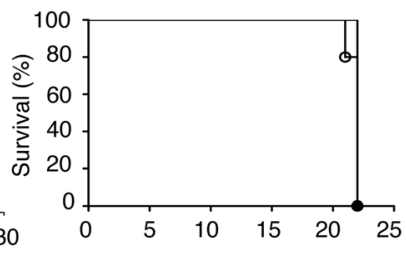

FIGURE 7 Vaccination with EVs or with recombinant HRF and PbEF-1a protect mice against parasite infection. SW (a) and C57BL/6 (b) mice were immunised with EVs and then infected with PbNK65 SPZ. In another set of experiments, C57BL/6 mice were infected with rPbEF-1a (c), rPbHRF (d), a mixture of rPbHRF and rPbEF-1a (e), or with EVs from uninfected RBCs (f) and then infected with $10^{4} \mathrm{PbNK} 65 \mathrm{SPZ}$. Parasitemia and survival (log-rank test, $P=0.01$ [a], $P=0.04$ [b], $P=0.0027$ [c], $P=0.00027$ [e]) were monitored over time. Insert in each panel highlights the difference in parasitemia between immunised and control mice from Day 3 to Day 10, which was log transformed for parasitemia normalisation before statistical analysis. Control groups (CTL) consisted of mice immunised with adjuvant alone. N.I. threshold of parasitemia detection. Error bars, SEM. Data are representative of two independent experiments with five mice per group; ${ }^{*} 0.0268<P<0.0345,{ }^{* *} 0.0079<P<0.0159$, Mann-Whitney test

The presence of EF-1a within PbEVs is reminiscent of similar observations made in Leishmania parasites. Indeed, two parasite proteins GP63 and EF-1a, known to activate the host protein-tyrosine phosphatases, which dephosphorylate key targets in multiple signalling pathways (MAP kinases/NF-KB pathway), were identified within Leishmania-derived exosomes. Moreover, it was shown that Leishmania EF-1a activated SHP-1 both in vitro and in vivo and that its delivery to macrophages prevented the induction of iNOS in response to treatment with IFN- $\gamma$ (Nandan et al., 2002), thus limiting their phagocytic function. Functionally, similar to the HRF protein, EF-1acontaining EVs were also found to inhibit the DTH response whereas EF-1a-deficient EVs lost this activity, indicating again that EF-1a acts also as an immunomodulatory factor that targets $T$ cell functions in an antigen-dependent manner.

Because HRF and EF-1a can both be considered as parasite virulence factors and because mutant parasites for both genes have similar phenotypes, we propose that these two parasite proteins may act in concert or interact synergistically with each other to interfere with the host $\mathrm{T}$ cell response. This scenario may explain why deleting either of the two proteins resulted in a similar loss of $\mathrm{T}$ cell inhibiting activity. Interestingly, a study focusing on the characterisation of HRF function using a yeast two-hybrid hunt identified the translation elongation factor, eEF-1a, and its guanine nucleotide exchange factor, eEF-1B $\beta$, as HRF-interacting partners (Cans et al., 2003; Langdon, Vonakis, \& MacDonald, 2004). Given the above, we hypothesise that malaria parasites secrete EVs to manipulate $T$ cell responses by transferring active proteins such as HRF and EF-1a and that their packaging within EVs ensures their protection and their long range activities. These findings are consistent with the fact that immunisation of mice with either protein conferred antiparasite protection and survival lower than when mice were immunised with a mixture of both proteins (Figure 7).

Mechanistically, we report here that T cells incubated in the presence of PbEVs during TCR-mediated activation displayed inhibition of various activation signalling pathways, as assessed by the reduced phosphorylation of key proteins such as PLC $\gamma 1$, Akt, and Erk and diminished production of cytokines. Interestingly, PbEVs from HRF $\triangle$ 
PbNK65 parasites have no significant inhibitory effects, indicating again the primary function of HRF associated to EVs in inducing immune suppression at the level of T cells. Reports in tumour immunology pointed out similar immunosuppressive effects on antitumour-specific $T$ cells by tumour-derived exosomes by inhibiting various activation endpoints including translocation of NFKB and NFAT into the nucleus, upregulation of CD69 and CD107a, production of cytokines, and cell proliferation (Shenoy et al., 2018). Alternatively, tumour-derived exosomes possess diverse immunosuppressive properties, such as negatively regulating the function of APCs and promoting the function of regulatory T cells (Clayton, Mitchell, Court, Mason, \& Tabi, 2007; Taylor \& Gercel-Taylor, 2005; Taylor, GercelTaylor, Lyons, Stanson, \& Whiteside, 2003; Yu et al., 2007).

Release of EVs by parasites and their immunomodulatory effects on host immune cells offers new opportunities to design preventive measures to control malaria disease. In this regard, immunisation of mice with EVs protected mice with more or less efficiency depending on their genetic background, conferring more protective efficacy to outbred Swiss mice with survival of $80 \%$ of mice. Remarkably, immunisation of mice with either $\mathrm{rPbEF}-1 a$ alone or in combination with $\mathrm{r} P b \mathrm{HRF}$ protected mice against SPZ challenge, and after parasite clearance, mice became completely parasite-free after a subsequent sprozoite infection, thus acquiring a long-lasting immune memory. This is very encouraging, considering the human perspective because preliminary results indicate that $P$. falciparum NF54 strain derived EVs also harbour HRF and EF-1a.

In conclusion, we demonstrate that parasite-derived EVs can act as immunosuppressive vesicles that target $T$ cells, adding a new layer of immune evasion mechanisms to the existing repertoire. We identify two conserved parasite components that interfere with the T cell signalling pathway resulting in non-functional immune responses. Our work has identified a new pathway that could be targeted for enhancing protective immunity against Plasmodium parasite infections. The development of strategies based on recombinant malaria vaccines could benefit from this work to overcome EV-mediated immune suppression in malaria-infected individuals.

\section{4 | EXPERIMENTAL PROCEDURES}

\section{1 | Ethics statements}

All animal care and experiments involving mice and immunisation of rabbits described in the present study strictly followed the institutional guidelines and the European Communities recommendations. This study was approved by the "Direction Départementale des Services Vétérinaires" de Paris, France (permit no. 75-066 issued on September 14, 2009) and the French Ministry of Higher Education and Research (project no. 00218.01).

\section{2 | Rodents}

Five- to 8-week-old wild-type female C57BL/6J Rj and SW mice were purchased from Janvier laboratory (Le Genest-Saint-Isle, France) and were used for experimental infections and EVs functional studies. The OT-2 transgenic mice, expressing a transgenic TCR that recognises the $\mathrm{OVA}_{323-339}$ peptide in the context of I-A $\mathrm{A}^{\mathrm{b}}$ (Barnden, Allison, Heath, \& Carbone, 1998), were bred in our animal facility. All mice were housed in the Central Animal Facility of the Institut Pasteur, under specific pathogen-free conditions.

\section{3 | Parasite lines}

Mice were inoculated with red blood cells infected (iRBCs) with either green fluorescent protein-transgenic $P$. berghei NK65 (MRA-268) or $P$. berghei ANKA (MRA-867) parasites and with their hrf (hrf $\triangle$ ), EF-1a (eEF-1aa $\Delta$ or eEF-1ab $\Delta$; Janse et al., 2003), and hmgb2 (hmgb2 $\Delta$ )deficient counterpart.

\subsection{Antibodies}

Purified mAbs used for flow cytometry were fluorescein isothiocyanate-labelled anti-CD45 (30-F11), phycoerythrin-labelled anti-IFN- $\gamma$, allophycocyanine-labelled anti-CD3 (145-2C11), Pacific Blue-labelled anti-CD8a (53-6.7), and AlexaFluor 700-labelled antiCD4 (RM4-5) antibodies (all antibodies from BD Biosciences, Mountain View, CA). The following reagents were used for cell stimulation, immunoblotting, or immunoprecipitation: anti-CD3-biontinylated, anti-CD28-biontinylated (eBiosciences, San Diego, CA), streptavidin (Sigma, St. Louis, MO); anti-pErk1/2 (pT202/pY204), anti-pPLCY1 (pY783), anti-pAkt (pS473; Cell Signaling Technology, Beverly, MA), rabbit anti-PbHRF, mouse anti-PbMSP1 $1_{1-33}$, rabbit anti-PbHMGB2, rabbit anti-PfEF-1a, and mouse anti-PfHSP70.

\section{5 | Mouse infections}

Cryopreserved $P$. berghei parasites were passaged once through SW mice before being used to infect experimental animals. Mice were infected with $10^{5}$ iRBCs injected intraperitoneally (i.p.) or by injecting $10^{4} \mathrm{SPZs}$ intravenously (i.v.). After the infection, blood samples were taken daily from the tail, and the parasitemia was assessed by flow cytometry and the results expressed in percentage of iRBCs.

\subsection{Ex vivo culture of $P$. berghei iRBCs}

When parasitemia reached $8-10 \%$ donor, mice blood was collected by intracardiac puncture under anaesthesia (Imalgene ${ }^{\circledR} 1000$ [Merial] and Rompun ${ }^{\circledR} 2 \%$ [Bayer]) in heparin at a dilution 1/10 (Sigma-Aldrich). To isolate the erythrocytes, blood was centrifuged at $400 \mathrm{~g}$ for $10 \mathrm{~min}$ at room temperature. The plasma supernatant was removed, and the cell fraction was washed by adding sterile PBS $1 \times$. After centrifugation at $400 \mathrm{~g}$ for $10 \mathrm{~min}$ at room temperature, the supernatant was aspirated, and the cell fraction was gently bringing back into suspension in prewarmed buffer RPMI1640 (Gibco, Thermo Fisher Scientific, Rockford, IL; pH 7.3) supplemented with 25 mM HEPES (Gibco, Thermo Fisher Scientific), $10 \mathrm{mM}$ sodium bicarbonate (Sigma-Aldrich), $25 \mathrm{U} / \mathrm{L}$ 
Néomycine (Sigma-Aldrich), and 10\% exosomes free fetal calf serum (Gibco, Thermo Fisher Scientific). Contamination of P. berghei iRBCs with host leukocytes and platelets was removed using custom-made CF11 cellulose powder (Cellulose C6288 from Sigma-Aldrich) columns. Purified erythrocytes were cultured into a $500 \mathrm{ml}$ Erlenmeyer flask for 12-16 hr on a shaker at $37^{\circ} \mathrm{C}$ under gas mixture: $5 \% \mathrm{CO}_{2}$ and $10 \% \mathrm{O}_{2}$ in $50 \mathrm{ml}$ (for $1.5 \mathrm{ml}$ pellet add $50 \mathrm{ml}$ of medium) of RPMI1640 complete exosomes free culture medium.

\section{7 | Isolation of Plasmodium-derived EVs}

EVs from supernatants of cultured $P$. berghei iRBCs were isolated by sequential centrifugations and ultracentrifugations. Briefly, ex vivo Plasmodium cultures were first transferred to a $50 \mathrm{ml}$ tube and centrifuged at $400 \mathrm{~g}$ for $10 \mathrm{~min}$ at $4^{\circ} \mathrm{C}$ for the elimination of erythrocytes. The harvested supernatant was further centrifuged at 2,000 $\mathrm{g}$ for $10 \mathrm{~min}$ at $4^{\circ} \mathrm{C}$ to get rid of residual cells and debris. EVs were isolated from the obtained supernatant by sequential ultracentifugations at $15,000 \mathrm{~g}$ for $2 \mathrm{hr}$ at $4^{\circ} \mathrm{C}$ (Beckman 45 Ti rotor; Beckman Coulter, Brea, $\mathrm{CA})$ to get rid of microvesicles, and EVs were finally pelleted at 150,000 g (Beckman $45 \mathrm{Ti}$ rotor; Beckman Coulter) for $3 \mathrm{hr}$ at $4^{\circ} \mathrm{C}$ to obtain. After the first ultracentrifugation step, and in order to eliminate large cell debris and membranes, the supernatant was filtered through a $0.1 \mu \mathrm{m}$ membrane (Millex V filter, Merck Millipore, Billerica, MA). To remove possible protein contaminations, EVs pellet was washed in a large volume of PBS and ultracentrifugated at 150,000 g (Beckman 70.1 Ti; Beckman Coulter) for $2 \mathrm{hr}$ at $4^{\circ} \mathrm{C}$. The final pellet was resuspended in $100 \mu \mathrm{l}$ of PBS and maintained on ice or stored at $-80^{\circ} \mathrm{C}$. For cell stimulation assays, freshly isolated vesicles were used. Protein concentrations were determined using BCA assay (Pierce) according to manufacturer's instructions.

\section{8 | Parasite extracts}

Parasite pellets obtained by saponin lysis and derived EVs from asynchronous asexual stages were incubated in lysis buffer $(50 \mathrm{mM}$ Tris, $\mathrm{pH}$ 8.0, $300 \mathrm{mM} \mathrm{NaCl}, 0.1 \mathrm{mM}$ EDTA, 0.5\% Triton, 1× protease inhibitors cocktail [Roche]) for $30 \mathrm{~min}$ on ice, briefly sonicated and spun at $14,000 \mathrm{rpm}$ for $10 \mathrm{~min}$ at $4^{\circ} \mathrm{C}$.

\subsection{Purification of recombinant PbHRF and PbEF-1a}

A pRSET A (Life Technologies Paisley, United Kingdom) plasmid containing the DNA encoding for the PbNK65 HRF was obtained from Kim Taylor (RMIT University, Australia), and Escherichia coli BL21 was used for expression. PbEF-1a was PCR amplified using the following oligos: Xhol-R: GTGGTGGTGGTGCTCGAGTTATTITITGCTGGTGC and Ndel-F: CCGCGCGGCAGCCATATGATGGGAAAAGAAAAAACTCAC. The PCR products were digested with $\mathrm{Xhol}$ and Ndel restriction enzymes and ligated into pET28b expression vector (Novagen). The positive clones were transformed in BL21 Star (DE3) E. coli strain
(Stratagene). Bacteria grew in a $10 \mathrm{ml}$ and $200 \mathrm{ml}$ overnight preculture respectively in Luria broth (LB), with specific antibiotics, at $37^{\circ} \mathrm{C}$. $\mathrm{PbHRF}$ and $\mathrm{PbEF}-1 \mathrm{a}$ expression was performed by diluting the preculture (1/10) in $500 \mathrm{ml}$ of $\mathrm{LB}$ kept at $37^{\circ} \mathrm{C}$ until the $\mathrm{OD}_{600}$ was 0.5 and induced for $3 \mathrm{hr}$ with $1 \mathrm{mM}$ IPTG, and $1 \mathrm{~L}$ of NZY Auto-Induction LB medium (NZYTech, Lda) incubated under agitation at $200 \mathrm{rpm}$ first for $4 \mathrm{hr}$ at $37^{\circ} \mathrm{C}$ followed by $15 \mathrm{hr}$ culture at $16^{\circ} \mathrm{C}$, respectively. The bacteria pellet was collected by centrifugation and resuspended in $50 \mathrm{mM}$ Tris- $\mathrm{HCl} \mathrm{pH} 7.5$, containing $150 \mathrm{mM} \mathrm{NaCl}$, $10 \mathrm{mM}$ imidazole, $1 \mathrm{mM}$ DTT, $2 \mathrm{mM} \mathrm{MgCl}_{2}$, anti-proteases (Roche), and benzonase (Sigma). The pellet was homogenised in a blender (Waring Commercial), submitted to cell disintegrator at $2.7 \mathrm{KBar}$ (Constant System TS) and centrifuged at 20,000 g for $1 \mathrm{hr}$ at $4^{\circ} \mathrm{C}$ in a SS34 rotor of a Sorvall centrifuge. For purification of (His) ${ }_{6}$-PbHRF or $\mathrm{PbEF}-1 \mathrm{a}, 100 \mathrm{ml}$ of the soluble fraction was loaded on a $5 \mathrm{ml}$ Protino Ni-NTA (Macherey-Nagel) column using ÄKTA Express chromatography system at $4^{\circ} \mathrm{C}$. After washes, first in equilibration buffer containing $0.5 \%$ Triton $\mathrm{X} 114$ for endotoxin removal and then in equilibration buffer, the protein was eluted with $50 \mathrm{mM}$ Tris- $\mathrm{HCl} \mathrm{pH} \mathrm{7.5,500} \mathrm{mM}$ $\mathrm{NaCl}, 500 \mathrm{mM}$ imidazole, $1 \mathrm{mM}$ DTT, and $2 \mathrm{mM} \mathrm{MgCl}$. After profile analysis by SDS-polyacrylamide gel and Coomassie blue staining, the selected fractions were cleaved, and the whole mixture was loaded on a second Ni-NTA column under the same condition used previously. The pass-through containing the purified cleaved protein was collected and directed applied on a benzamidine sepharose column

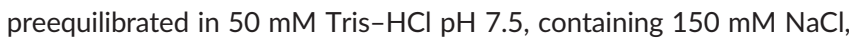
$10 \mathrm{mM}$ imidazole, $1 \mathrm{mM} \mathrm{DTT}$, and $2 \mathrm{mM} \mathrm{MgCl}_{2}$. The $\mathrm{rPbHRF}$ and $\mathrm{rPbEF}-1 \mathrm{a}$ purified protein was stored at $4^{\circ} \mathrm{C}$. The possible presence of protein aggregates was measured by DLS.

\subsection{Antibodies production and purification}

The purified rPbHRF (100 $\mu \mathrm{g}$ ) or the two PbEF-1a immunogenic peptide sequences, identified by using an algorithm, coupled to Keyhole limpet haemocyanin, were injected intradermally into the back skin of a rabbit in the presence of complete Freund's adjuvant followed by two booster injections in the presence of incomplete Freund's adjuvant. Rabbit IgG antibodies were isolated using a protein A column as an immunoabsorbent. Rabbit anti-PbEF-1a was obtained from J. Hans Kroeze, Department of Parasitology, Center of Infectious Diseases, Leiden University Medical Center, whereas the rabbit anti-HRF was produced in-house specifically for the purposes of this study (Mathieu et al., 2015).

\subsection{1 | Mice immunisation}

C57BL/6J Rj and SW mice were immunised with $25 \mu \mathrm{g}$ of $P$. berghei NK65-derived EVs, rPbHRF, rPbEF-1a, or a mixture of rPbHRF and $\mathrm{rPbEF}-1 \mathrm{a}$ in the presence of complete Freund's adjuvant (Sigma) followed 2 weeks later by two booster injections at 1 week interval in the presence of incomplete Freund's adjuvant. Mice immunised with Freund's adjuvant served as controls. The formulation was 
administered intraperitoneally in a total volume of $100 \mu \mathrm{l}$. Sera were collected before the first immunisation (Day 0 , negative control), 2 weeks after the first immunisation, 1 week after the last immunisation and were tested of the presence of the antigen-specific antibodies. Immunised mice were then infected with $10^{4}$ PbNK65 SPZs. In experiments exploring the immune memory against parasites, the group of mice that received the PbNK65 after the immunisation and completely eliminated it from the blood stream were additionally challenged with $10^{4}$ PbNK65 SPZs.

\subsection{2 | Cell preparation}

Following sacrifice of mice by deep ether anaesthesia, spleen, lymph nodes, and bone marrow were removed. Briefly, tissues were removed and homogenised in complete exosome free RPMI 1640 medium (Gibco, Thermo Fisher Scientific) by passage through sterile meshes to obtain a single-cell suspension. To remove contaminating erythrocytes if present, the cell suspensions were subjected to osmotic lysis using a hypotonic ammonium chloride solution. In the preparation of mature murine DCs, $1 \times 10^{6} / \mathrm{ml}$ immature cells, derived from C57BL/6 mice bone marrow, were plated and placed in tissue culture incubator. Cells were cultured as previously described (Matheu, Sen, Cahalan, \& Parker, 2008). After 10-11 days of culture, the mature murine DCs were harvested and used in proliferation assays.

\subsection{3 | Proliferation assays}

For proliferation analysis by CFSE method, cells were labelled with CFSE (CellTrace; Gibco, Thermo Fisher Scientific) for $10 \mathrm{~min}$ at $37^{\circ} \mathrm{C}$, previous to stimulation, for assessment of cell proliferation. Labelled cells were washed, then resuspended in culture media at $0.5 \times 10^{6}$ cells $/ \mathrm{ml}$. Cells were cultured in 96 -well plates $37^{\circ} \mathrm{C}$ in $5 \% \mathrm{CO}_{2} 24$, 48,72 , and $96 \mathrm{hr}$ after being stimulated by addition of ConA and OVA antigens or using anti-CD3/CD28 antibodies (Dynabeads Mouse T-Activator; Gibco, Thermo Fisher Scientific). Cells were stained with anti-CD8 and anti-CD4 20 min at $25^{\circ} \mathrm{C}$, washed in PBS with $0.01 \%$ $\mathrm{BSA}$, then analysed by flow cytometric.

\subsection{4 | OVA-induced DTH reaction at footpad}

To induce a DTH reaction, 5- to 8-week-old wild-type female C57BL/6J mice were immunised with 50 mg OVA (Grade V; SigmaAldrich) emulsified in $25 \mu \mathrm{l}$ complete Freund's adjuvant (Sigma-Aldrich) by subcutaneous injection at the base of the tail. Seven days later, $30 \mu \mathrm{l}$ of $2 \%$ aggregated OVA (Grade II) was subcutaneously injected into the centre of the right hind footpad and $30 \mu \mathrm{l}$ of saline in the other as a control. The thickness of the footpad was measured with callipers at the indicated time points $(24,48$, and $72 \mathrm{hr}$ postchallenge) by subtracting values given by saline-injected footpads from those of Ag-injected footpads. Aggregated OVA was prepared by heating a $2 \%$ solution of OVA at $70^{\circ} \mathrm{C}$ for $1 \mathrm{hr}$. After cooling, the precipitate was washed and resuspended in the original volume of saline. For tissue section analysis, mice were euthanised at $24 \mathrm{hr}$ after aggregated OVA challenge.

\subsection{5 | Flow cytometry}

Single-cell suspension was stained for FACS analysis according to standard protocols in cold PBS containing $2 \%$ fetal calf serum and $0.01 \%$ sodium azide. Before staining, cells were preincubated $10 \mathrm{~min}$ on ice with the rat $\mathrm{mAb}$ antimouse CD16/CD32 (clone 2.4G2; BD Biosciences) to block Fc receptors, then incubated with the appropriate combination of fluorescent-labelled antibodies. In all experiment, 7amino actinomycin D (Sigma-Aldrich) was used to exclude dead cells from the analysis. Doublets were systematically excluded based on side scatter and forward scatter parameters. For intracellular staining, cells were stimulated by $\mathrm{PMA} /$ Iono for $4 \mathrm{hr}$ at $37^{\circ} \mathrm{C}$, and activation was stopped by adding paraformaldehyde at $4 \%$ final concentration. Flow cytometry experiments were performed using CytoFLEX S flow cytometer equipped with CyExpert 2.2.0.97 software (Beckman Coulter). Flow cytometry data were analysed using FlowJo v10.3.0 software (FlowJo, LLC, Ashland, OR).

\subsection{EV labelling and Amnis ImageStreamX analysis}

The EV suspension was mixed with a solution of TO (Sigma-Aldrich) diluted 1:500 in PBS and incubated for $30 \mathrm{~min}$ at $37^{\circ} \mathrm{C}$. Labelled EVs were washed with PBS and ultracentrifuged at 150,000 g (Beckman $45 \mathrm{Ti}$ rotor; Beckman Coulter) for $3 \mathrm{hr}$. Acquisition was performed using ImageStreamX Imaging Flow Cytometer (Amnis Corporation, Seattle, WA) equipped with INSPIRE software. A 60x magnification was used for all samples. A minimum of $5 \times 10^{5}$ lymph node cells, labelled with AlexaFluor 700 anti-CD4 and Pacific Blue anti-CD8a, were analysed for each sample. Data analysis was performed using the IDEAS v6 software (Amnis Corporation). TO, AlexaFluor 700, and Pacific Blue fluorescence were collected on Channel 2, Channel 11 , and Channel 7, respectively. Intensity-adjusted brightfield images were collected on Channel 1.

\subsection{7 | Immunofluorescence assays}

Fixation and permeabilisation infected RBCs was performed using 4\% paraformaldehyde and 0.01\% Triton X-100 and blocked with 3\% (w/v) gelatin from porcine skin in PBS (Sigma-Aldrich). Thereafter, cells were incubated with primary antibody for $1 \mathrm{hr}$ at RT; dilutions were 1:500 for anti-HRF (Mathieu et al., 2015) and anti-EF-1a, antibodies in 3\% $(\mathrm{w} / \mathrm{v})$ gelatin from porcine skin in PBS. After three washes with PBS, primary antibodies were detected with Alexa Fluor 568- or 647conjugated secondary antibodies (Thermo Fisher Scientific) diluted $1: 2,000$ in $3 \%(w / v)$ gelatin from porcine skin in PBS. After three final washes in PBS, cells were mounted in ProLong Gold containing DAPI (Thermo Fisher Scientific) for nuclear staining. Images were captured using a Deltavision Elite imaging system (GE Healthcare). Image processing of at least two independent replicates was performed using 
the Fiji package (http://fiji.sc). To measure the amount of colocalisation between EF-1- $\alpha$ and HRF clusters and its statistical significance, we designed a multistep approach in Icy software (http:// icy.bioimageanalysis.org/; de Chaumont et al., 2012). First, we draw manually an ROI around green fluorescent protein signal in each RBC and segmented automatically HRF and EF-1-a clusters using wavelet transformation of the image and statistical thresholding (plugin Spot Detector in Icy, using scale 2 and threshold = 50; OlivoMarin, 2002). The overlap between segmented HRF and EF-1a masks was measured with Manders coefficients in Icy plugin Colocalization Studio (Lagache et al., 2015): M1 = Surface (Intersection between HRF \& EF-1a masks)/Surface (HRF masks) and M2 = Surface (Intersection between HRF \& EF-1a masks)/Surface (EF-1a masks) (Experimental values). To test the statistical significance of measured M1 and M2 coefficients, we then randomised 1,000 times the position of EF1a masks inside each ROI and computed the corresponding $\mathrm{M} 1$ and M2 coefficients (random values). Finally, we estimated the empirical $P$ value with the rank order of experimental M1 and M2 compared with simulations. We observed that measured $\mathrm{M} 1$ and $\mathrm{M} 2$ coefficients were in the last percentile of simulations indicating a significantly high overlap and molecule colocalisation.

\subsection{8 | Immunoprecipitation}

Immunoprecipitation of PbHRF-PbEF-1a complex was performed using the Pierce Direct Immunoprecipitation Kit (Thermo Fisher Scientific). Before immunoprecipitation, $10 \mu \mathrm{g}$ of purified anti-HRF or antiEF-1a antibodies was directly immobilised onto an agarose support (AminoLink Plus Resin) using a short coupling protocol; 100 mg of parasite extracts or recombinant proteins was incubated with the immobilised antibody to form the immune complex with gentle endover-end mixing overnight at $4^{\circ} \mathrm{C}$. To remove nonbound material, beads were washed three times with wash buffer, and a low $\mathrm{pH}$ elution buffer was used to dissociate the bound antigen from the antibody. Immunoprecipitated proteins were then analysed by western blotting.

\subsection{9 | DLS analysis}

Control and Plasmodium-derived EVs samples were diluted 1:4 in PBS, and DLS analysis was performed on DynaPro Plate Reader (Wyatt, Santa Barbara, CA). Data were recorded at $20^{\circ} \mathrm{C}$ monitored using dynamics V7 software (Wyatt)

\subsection{0 | TRPS analysis}

Size distribution, diameter, and concentration of Plasmodium-derived EVs were quantified by TRPS using the qNano (Izon Science, Oxford, UK). All samples were diluted 1:4 in PBS prior to the measurement. NP200 (Izon Science) pores and a pressure of 7 or 8 mbar were used. Calibration runs for voltage, stretch, pressure, and baseline current were performed with two standard beads (114 and $70 \mathrm{~nm}$ diameter carboxylated polystyrene beads). Data analysis was performed by qNano IZON software (Izon Control Suite, version 3.2, Izon Science).

\subsection{Nanoparticle tracking analysis}

The size and concentration of EVs were analysed using the NanoSight NS300 system (Malvern Instruments, Malvern, UK) with 488 nm laser, sCMOS camera module, and NTA software version 3.2. For NanoSight analysis, EVs were diluted in $1 \mathrm{ml}$ of PBS; each sample was recorded five times for $60 \mathrm{~s}$ in static mode at constant temperature of $25^{\circ} \mathrm{C}$ creating five replicable histograms, which were averaged.

\subsection{2 | Atomic force microscopy}

Exosome extracts were diluted by 100x in PBS before deposition. The diluted sample was incubated on freshly cleaved mica for $5 \mathrm{~min}$, then rinsed with $350 \mu \mathrm{l}$ aliquots of PBS. The remaining liquid was immediately absorbed by aspirating to an absorbent filter paper held at the sample edge, and then the sample was dried under a gentle dry nitrogen flow. AFM measurements were made on a Multimode AFM (Bruker, Santa Barbara, CA) using either a custom fast scanning AFM head (Adams et al., 2014) in tapping mode or in ScanAsyst ${ }^{\mathrm{rm}}$ mode. Fast-Scan B probes (Bruker) were used for the former and PNP-TRS probes (Nanoworld, Neuchatel, Switzerland) for the latter.

\subsection{3 | MS analysis}

\subsection{1 | Sample preparation}

Each shaved-pellet sample was lysed in $50 \mu \mathrm{l}$ lysis buffer (2\% SDS, $50 \mathrm{mM}$ Tris $\mathrm{pH}$ 8.5, $20 \mathrm{mM}$ TCEP, $50 \mathrm{mM}$ chloroacetamide) and heated $5 \mathrm{~min}$ at $95^{\circ} \mathrm{C}$. Proteins were trypsin-digested overnight using the filtered-aided sample preparation method, as described (Wisniewski, Zougman, Nagaraj, \& Mann, 2009). Filtered peptides were desalted on $\mathrm{C}_{18}$ reverse-phase stageTips, eluted, and dried down using a speed vac concentrator (Millipore, Burlington, MA).

\subsection{2 | Bottom-up LC-MS/MS analysis}

Peptides were concentrated and separated by nano high-performance liquid chromatography (nanoflow LC-MS/MS) hyphenated with a mass spectrometer: $1 \mu \mathrm{l}$ of each sample was analysed using an RSLC Ultimate 3000 Liquid Chromatographic system coupled to a hybrid Q Exactive plus mass spectrometer (both from Thermo Fisher Scientific). Briefly, peptides were loaded and washed on a $C_{18}$ reversephase precolumn ( $3 \mu \mathrm{m}$ particle size, $100 \AA$ pore size, $75 \mu \mathrm{m}$ i.d., $2 \mathrm{~cm}$ length; Thermo Fisher Scientific). The loading buffer contained 98\% $\mathrm{MilliQH}_{2} \mathrm{O}, 2 \%$ acetonitrile, and 0.1\% TFA. Peptides were then separated on a $\mathrm{C}_{18}$ reverse-phase resin ( $2 \mu \mathrm{m}$ particle size, $100 \AA$ pore size, $75 \mu \mathrm{m}$ i.d.,25 cm length from Thermo Fisher Scientific) with a 35 min "effective" gradient from 99\% A $(0.1 \%$ formic acid and 100\% $\mathrm{H}_{2} \mathrm{O}$ ) to $40 \% \mathrm{~B}$ (80\% acetonitrile, $0.085 \%$ formic acid and $20 \% \mathrm{H}_{2} \mathrm{O}$ ). The mass spectrometer acquired data throughout the elution process 
and operated in a data-dependent scheme with full MS scans acquired in the orbitrap analyser, followed by up to $10 \mathrm{MS} / \mathrm{MS}$ higher energy collisional dissociation spectra on the most abundant ions detected in the MS scan. Mass spectrometer settings were full MS (AGC: 3.10e6, resolution: 70,000, m/z range 400-2,000, maximum ion injection time: $100 \mathrm{~ms}$ ); MS/MS (normalised collision energy: 30, resolution: 17,500 , intensity threshold: $1.10 \mathrm{e} 4$, isolation window: $4.0 \mathrm{~m} / \mathrm{z}$, dynamic exclusion time setting: $15 \mathrm{~s}$, AGC target: $1.10 \mathrm{e} 5$ and maximum ion injection time: $100 \mathrm{~ms}$ ). The fragmentation was permitted for precursor with a charge state of two to four excluding isotopes.

\subsection{3 | Database queries with extracted experimental data}

Two FASTA-formatted protein databases were used: (a) the SwissProt database release 2017-06 (554,860 sequences) and (b) the Plasmodium sp database extracted from NCBInr 2017-01 (397,800 sequences). Extracted peptides fragment mass lists were compared with the above databases using Maxquant version 1.6.1 (Cox \& Mann, 2008). The enzyme specificity was trypsin's. Cysteins carbamidomethylation was considered complete whereas variable modifications were restricted to protein $\mathrm{N}$-terminal acetylation and methionine oxidation. The peptide tolerance was set to $4.5 \mathrm{ppm}$ and the fragment mass tolerance to $20 \mathrm{ppm}$. Minimal length of peptides was set at seven amino acids and maximal length at 6,000 $\mathrm{Da}$. Second peptide search was allowed. False discovery rate was kept below $1 \%$ on both peptide and protein levels. Label-free protein quantification was done using both unique and razor peptides (Cox et al., 2014) with at least two ratio counts. Data from all experiments were analysed simultaneously. Proteins' signal intensity was converted to ppm relative to the total signal intensity of each replicate preparation (Sum of protein-related peptide intensities in a sample/sum of all peptide intensities of all proteins detected in the sample $\times 1.10 \mathrm{e} 6$ ) to account for relative intensity of a specific protein across the five replicates.

\subsection{ELISA detection of specific antibodies and cytokines}

Humoral responses elicited by EVs, rPbHRF, rPbEF-1a, or a mixture of $\mathrm{rPbHRF}$ and $\mathrm{rPbEF}-1 \mathrm{a}$ during the immunisation were studied by ELISA. Parasite-derived EVs and recombinant proteins at $1 \mu \mathrm{g} / \mathrm{ml}$ in carbonate buffer were coated overnight at $4^{\circ} \mathrm{C}$ onto 96 -well plates (Nuncimmuno plate; Thermo Scientific) and then blocked for $1 \mathrm{hr}$ at $37^{\circ} \mathrm{C}$ with a saturation buffer (PBS, 0.05\% tween, 3\% BSA). Sera samples from immunised mice were serially diluted and incubated on plates for $1 \mathrm{hr}$ at $37^{\circ} \mathrm{C}$. After washing steps (0.05\% tween in PBS), a secondary horseradish peroxidase conjugated goat antimouse lg antibody (Cell Signalling Technology ${ }^{\circledR}$, Danvers, MA) was added at a dilution of $1 / 2,000$ for $45 \mathrm{~min}$ at $37^{\circ} \mathrm{C}$. Antibody binding was revealed by addition of $o$-phenylenediamine dihydrochloride substrate (SigmaAldrich). Hydrogen chloride $(\mathrm{HCl}) 1 \mathrm{~N}$ was used to block the reaction. The optical density was read at 490-655 nm. Amounts of IFN- $\gamma$ and
IL-2 in the medium were analysed by cytokine-specific ELISA kits (BD Biosciences).

\subsection{5 | Western blotting}

Samples were either stored at $-20^{\circ} \mathrm{C}$ or directly fractionated by electrophoresis on SDS-PAGE gel (4-12\% Bis-Tris gels, BOLT mini gel system, Thermo Fisher Scientific) using MES buffer, then transferred to nitrocellulose membranes and analysed by immunoblotting. After blocking, membranes were incubated overnight at $4^{\circ} \mathrm{C}$ with primary antibodies. After several washes, blots were incubated with appropriate secondary antibodies for $45 \mathrm{~min}$ at room temperature. Immunoblots were detected by either enhanced chemiluminescence or by near-infrared fluorescence. In the first case, HRP-conjugated goat antirabbit IgG (1:10,000; Bio-Rad Laboratories, Hercules, CA) and antirabbit IgG (1:10,000; Cell Signaling Technology) were used, and specific bands were detected by chemoluminescence using the SuperSignal West Pico kit (Thermo Fisher Scientific) according to the manufacturer's instructions using ImageLab Touch software 1.2.0.12 (Bio-Rad Laboratories). In the latter case, secondary antibodies labelled with either AlexaFluor 680 (Invitrogen) or IRDye 800 (Rockland Immunochemicals) were used. Fluorescent immunoblot images were acquired and quantified by using an Odyssey scanner and the Odyssey 1.2 software (Li-Cor Biosciences, Inc., Lincoln, NE).

\subsection{6 | Effect of Plasmodium-derived EVs on the phosphorylation of proteins associated with TCR signalling}

Total lymph node cells from C57BL/6J mice were isolated and stimulated by anti-CD3 plus anti-CD28 antibody crosslinking for the indicated time points. Cells were subsequently lysed and analysed by immunoblotting using the indicated antibody. Images were acquired with an Odyssey infrared scanner (Li-Cor Biosciences, Inc.) and quantified. For each phosphoprotein, band intensities were quantified and normalised by the GPDH relative amount in the same lane. Normalised intensities were then divided by the mean normalised intensity of the same experiment.

\subsection{7 | Statistical analysis}

All data were analysed using GraphPad Prism 5.0 software. Unpaired data between two groups at a specific time point were analysed by Mann-Whitney test for nonparametric analysis when data did not fit a Gaussian distribution. A $P$ value of $<0.05$ was considered to be statistically significant. All experiments were replicated several times as indicated in the figure legends.

\section{ACKNOWLEDGEMENTS}

We thank Peter Smooker and Kim Taylor (Department of Biotechnology and Environmental Biology, RMIT University, Bundoora, Australia) for providing $P$. berghei HRF plasmid, Patty Chen (BIHP, Institut 
Pasteur, Paris) for her technical assistance on molecular biology, Bertrand Raynal (Platform of Molecular Biophysics, Institut Pasteur, Paris) for DLS analysis and technical assistance, Anthony Bouillon (Structural Biology Unit, Institut Pasteur, Paris) for the recombinant protein PbMSP1 and anti-MSP1 antibody, Mireille Nowakowski (Platform of recombinant proteins, Institut Pasteur, Paris) for preparing recombinant HRF and EF-1a proteins, and Evangéline Bennana (3P5 proteomics Facility of the Université Paris Descartes, Institut Cochin, Paris, France) for her technical assistance for mass spectrometry. We also thank the CEPIA (Centre d'élevage, de production et d'infection des anopheles, Institut Pasteur, Paris) for providing infected Anopheles mosquitoes.

\section{FUNDING INFORMATION}

This work has been supported by the French Parasitology consortium ParaFrap (ANR-11-LABX0024), by a collaborative research grant from the Pasteur-Weizmann joint research program, and by a grant from Institut Pasteur to S. M. C. D. G. was supported by a postdoctoral fellowship from the Helmut Horten Foundation, Agno, Switzerland. N. R.-R. is grateful for the support from the European Research Council (ERC) under the European Union's Horizon 2020 research and innovation program (Grant Agreement 757743), and the Israel Science Foundation (ISF) (619/16 and 119034).

\section{CONFLICTS OF INTEREST}

The authors declare no competing financial interests.

\section{ORCID}

Salaheddine Mécheri (D) https://orcid.org/0000-0003-3597-8810

\section{REFERENCES}

Abdi, A., Yu, L., Goulding, D., Rono, M. K., Bejon, P., Choudhary, J., \& Rayner, J. (2017). Proteomic analysis of extracellular vesicles from a Plasmodium falciparum Kenyan clinical isolate defines a core parasite secretome. Wellcome Open Research, 2, 50. https://doi.org/10.12688/ wellcomeopenres.11910.2

Adams, J. D., Nievergelt, A., Erickson, B. W., Yang, C., Dukic, M., \& Fantner, G. E. (2014). High-speed imaging upgrade for a standard sample scanning atomic force microscope using small cantilevers. The Review of Scientific Instruments, 85(9), 1-7, 093702. https://doi.org/10.1063/ 1.4895460

Amzallag, N., Passer, B. J., Allanic, D., Segura, E., Thery, C., Goud, B., ... Telerman, A. (2004). TSAP6 facilitates the secretion of translationally controlled tumor protein/histamine-releasing factor via a nonclassical pathway. The Journal of Biological Chemistry, 279(44), 46104-46112. https://doi.org/10.1074/jbc.M404850200

Bachmann, A., Esser, C., Petter, M., Predehl, S., von Kalckreuth, V., Schmiedel, S., ... Tannich, E. (2009). Absence of erythrocyte sequestration and lack of multicopy gene family expression in Plasmodium falciparum from a splenectomized malaria patient. PLoS ONE, 4(10), 1-6, e7459. https://doi.org/10.1371/journal.pone.0007459

Baglio, S. R., van Eijndhoven, M. A., Koppers-Lalic, D., Berenguer, J., Lougheed, S. M., Gibbs, S., ... Pegtel, D. M. (2016). Sensing of latent EBV infection through exosomal transfer of 5'pppRNA. Proceedings of the National Academy of Sciences of the United States of America, 113(5), E587-E596. https://doi.org/10.1073/pnas.1518130113

Barnden, M. J., Allison, J., Heath, W. R., \& Carbone, F. R. (1998). Defective TCR expression in transgenic mice constructed using CDNA-based alpha- and beta-chain genes under the control of heterologous regulatory elements. Immunology and Cell Biology, 76(1), 34-40. https://doi. org/10.1046/j.1440-1711.1998.00709.x

Bereczky, S., Dolo, A., Maiga, B., Hayano, M., Granath, F., Montgomery, S. M., ... Farnert, A. (2006). Spleen enlargement and genetic diversity of Plasmodium falciparum infection in two ethnic groups with different malaria susceptibility in Mali, West Africa. Transactions of the Royal Society of Tropical Medicine and Hygiene, 100(3), 248-257. https:// doi.org/10.1016/j.trstmh.2005.03.011

Briquet, S., Lawson-Hogban, N., Boisson, B., Soares, M. P., Peronet, R., Smith, L., ... Vaquero, C. (2015). Disruption of parasite hmgb2 gene attenuates Plasmodium berghei ANKA pathogenicity. Infection and Immunity, 83(7), 2771-2784. https://doi.org/10.1128/IAI.03129-14

Cans, C., Passer, B. J., Shalak, V., Nancy-Portebois, V., Crible, V., Amzallag, N., ... Telerman, A. (2003). Translationally controlled tumor protein acts as a guanine nucleotide dissociation inhibitor on the translation elongation factor eEF1A. Proceedings of the National Academy of Sciences of the United States of America, 100(24), 13892-13897. https://doi.org/ $10.1073 /$ pnas. 2335950100

Clayton, A., Mitchell, J. P., Court, J., Mason, M. D., \& Tabi, Z. (2007). Human tumor-derived exosomes selectively impair lymphocyte responses to interleukin-2. Cancer Research, 67(15), 7458-7466. https://doi.org/ 10.1158/0008-5472.can-06-3456

Couper, K. N., Barnes, T., Hafalla, J. C., Combes, V., Ryffel, B., Secher, T., ... de Souza, J. B. (2010). Parasite-derived plasma microparticles contribute significantly to malaria infection-induced inflammation through potent macrophage stimulation. PLoS Pathogens, 6(1), 1-13, e1000744. https://doi.org/10.1371/journal.ppat.1000744

Cox, J., Hein, M. Y., Luber, C. A., Paron, I., Nagaraj, N., \& Mann, M. (2014). Accurate proteome-wide label-free quantification by delayed normalization and maximal peptide ratio extraction, termed MaxLFQ. Molecular \& Cellular Proteomics, 13(9), 2513-2526. https://doi.org/ 10.1074/mcp.M113.031591

Cox, J., \& Mann, M. (2008). MaxQuant enables high peptide identification rates, individualized p.p.b.-range mass accuracies and proteome-wide protein quantification. Nature Biotechnology, 26(12), 1367-1372. https://doi.org/10.1038/nbt.1511

De Chaumont, F., Dallongeville, S., Chenouard, N., Herve, N., Pop, S., Provoost, T., ... Olivo-Marin, J. C. (2012). Icy: An open bioimage informatics platform for extended reproducible research. Nature Methods, 9(7), 690-696. https://doi.org/10.1038/nmeth.2075

Demarta-Gatsi, C., Peronet, R., Smith, L., Thiberge, S., Menard, R., \& Mecheri, S. (2017). Immunological memory to blood-stage malaria infection is controlled by the histamine releasing factor (HRF) of the parasite. Scientific Reports, 7(1), 9129-9141. https://doi.org/10.1038/ s41598-017-09684-2

Demarta-Gatsi, C., Smith, L., Thiberge, S., Peronet, R., Commere, P. H., Matondo, M., ... Mecheri, S. (2016). Protection against malaria in mice is induced by blood stage-arresting histamine-releasing factor (HRF)deficient parasites. The Journal of Experimental Medicine, 213(8), 1419-1428. https://doi.org/10.1084/jem.20151976

van Dongen, H. M., Masoumi, N., Witwer, K. W., \& Pegtel, D. M. (2016). Extracellular vesicles exploit viral entry routes for cargo delivery. Microbiology and Molecular Biology Reviews, 80(2), 369-386. https://doi.org/ 10.1128/mmbr.00063-15

Feng, Z., Hensley, L., McKnight, K. L., Hu, F., Madden, V., Ping, L., ... Lemon, S. M. (2013). A pathogenic picornavirus acquires an envelope by 
hijacking cellular membranes. Nature, 496(7445), 367-371. https://doi. org/10.1038/nature12029

Greenwood, B. M., Groenendaal, F., Bradley, A. K., Greenwood, A. M., Shenton, F., Tulloch, S., \& Hayes, R. (1987). Ethnic differences in the prevalence of splenomegaly and malaria in The Gambia. Annals of Tropical Medicine and Parasitology, 81(4), 345-354. https://doi.org/ 10.1080/00034983.1987.11812130

Janse, C. J., Haghparast, A., Speranca, M. A., Ramesar, J., Kroeze, H., del Portillo, H. A., \& Waters, A. P. (2003). Malaria parasites lacking eef1a have a normal $\mathrm{S} / \mathrm{M}$ phase yet grow more slowly due to a longer $\mathrm{G} 1$ phase. Molecular Microbiology, 50(5), 1539-1551. https://doi.org/ 10.1046/j.1365-2958.2003.03820.x

Lagache, T., Sauvonnet, N., Danglot, L., \& Olivo-Marin, J. C. (2015). Statistical analysis of molecule colocalization in bioimaging. Cytometry. Part A, 87(6), 568-579. https://doi.org/10.1002/cyto.a.22629

Langdon, J. M., Vonakis, B. M., \& MacDonald, S. M. (2004). Identification of the interaction between the human recombinant histamine releasing factor/translationally controlled tumor protein and elongation factor1 delta (also known as eElongation factor-1B beta). Biochimica et Biophysica Acta, 1688(3), 232-236. https://doi.org/10.1016/j. bbadis.2003.12.007

Looareesuwan, S., Ho, M., Wattanagoon, Y., White, N. J., Warrell, D. A., Bunnag, D., ... Wyler, D. J. (1987). Dynamic alteration in splenic function during acute falciparum malaria. The New England Journal of Medicine, 317(11), 675-679. https://doi.org/10.1056/ nejm198709103171105

Malda, J., Boere, J., van de Lest, C. H., van Weeren, P., \& Wauben, M. H. (2016). Extracellular vesicles-New tool for joint repair and regeneration. Nature Reviews Rheumatology, 12(4), 243-249. https://doi.org/ 10.1038/nrrheum.2015.170

Mantel, P. Y., Hoang, A. N., Goldowitz, I., Potashnikova, D., Hamza, B., Vorobjev, I., ... Marti, M. (2013). Malaria-infected erythrocyte-derived microvesicles mediate cellular communication within the parasite population and with the host immune system. Cell Host \& Microbe, 13(5), 521-534. https://doi.org/10.1016/j.chom.2013.04.009

Martin-Jaular, L., Nakayasu, E. S., Ferrer, M., Almeida, I. C., \& Del Portillo, H. A. (2011). Exosomes from Plasmodium yoelii-infected reticulocytes protect mice from lethal infections. PLoS ONE, 6(10), 1-10, e26588. https://doi.org/10.1371/journal.pone.0026588

Matheu, M. P., Sen, D., Cahalan, M. D., \& Parker, I. (2008). Generation of bone marrow derived murine dendritic cells for use in 2-photon imaging. Journal of Visualized Experiments, 9(17), 542-558. https://doi.org/ $10.3791 / 773$

Mathieu, C., Demarta-Gatsi, C., Porcherie, A., Brega, S., Thiberge, S., Ronce, K., ... Mecheri, S. (2015). Plasmodium berghei histamine-releasing factor favours liver-stage development via inhibition of IL-6 production and associates with a severe outcome of disease. Cellular Microbiology, 17(4), 542-558. https://doi.org/10.1111/cmi.12382

Muesch, A., Hartmann, E., Rohde, K., Rubartelli, A., Sitia, R., \& Rapoport, T. A. (1990). A novel pathway for secretory proteins? Trends in Biochemical Sciences, 15(3), 86-88. https://doi.org/10.1016/09680004(90)90186-F

Nandan, D., Yi, T., Lopez, M., Lai, C., \& Reiner, N. E. (2002). Leishmania EF1alpha activates the Src homology 2 domain containing tyrosine phosphatase SHP-1 leading to macrophage deactivation. The Journal of Biological Chemistry, 277(51), 50190-50197. https://doi.org/10.1074/ jbc.M209210200

Ofir-Birin, Y., Abou Karam, P., Rudik, A., Giladi, T., Porat, Z., \& Regev-Rudzki, N. (2018). Monitoring extracellular vesicle cargo active uptake by imaging flow cytometry. Frontiers in Immunology, 9, 1-9. https://doi.org/10.3389/fimmu.2018.01011
Olivo-Marin, J.-C. (2002). Extraction of spots in biological images using multiscale products. Pattern Recognition, 35(9), 1989-1996. https:// doi.org/10.1016/S0031-3203(01)00127-3

Oomen, J. M., Meuwissen, J. H., \& Gemert, W. (1979). Differences in blood status of three ethnic groups inhabiting the same locality in Northern Nigeria. Anaemia, splenomegaly and associated causes. Tropical and Geographical Medicine, 31(4), 587-606.

Opperdoes, F. R., \& Szikora, J. P. (2006). In silico prediction of the glycosomal enzymes of Leishmania major and trypanosomes. Molecular and Biochemical Parasitology, 147(2), 193-206. https://doi.org/ 10.1016/j.molbiopara.2006.02.010

Ramakrishnaiah, V., Thumann, C., Fofana, I., Habersetzer, F., Pan, Q., de Ruiter, P. E., ... van der Laan, L. J. (2013). Exosome-mediated transmission of hepatitis $\mathrm{C}$ virus between human hepatoma Huh7.5 cells. Proceedings of the National Academy of Sciences of the United States of America, 110(32), 13109-13113. https://doi.org/10.1073/ pnas. 1221899110

Raposo, G., \& Stoorvogel, W. (2013). Extracellular vesicles: Exosomes, microvesicles, and friends. The Journal of Cell Biology, 200(4), 373-383. https://doi.org/10.1083/jcb.201211138

Record, M., Silvente-Poirot, S., Poirot, M., \& Wakelam, M. J. O. (2018). Extracellular vesicles: Lipids as key components of their biogenesis and functions. Journal of Lipid Research https://doi.org/10.1194/jlr. E086173, 59, 1316-1324.

Regev-Rudzki, N., Wilson, D. W., Carvalho, T. G., Sisquella, X., Coleman, B. M., Rug, M., ... Cowman, A. F. (2013). Cell-cell communication between malaria-infected red blood cells via exosome-like vesicles. Cell, 153(5), 1120-1133. https://doi.org/10.1016/j.cell.2013.04.029

Ridder, K., Sevko, A., Heide, J., Dams, M., Rupp, A. K., Macas, J., ... Momma, S. (2015). Extracellular vesicle-mediated transfer of functional RNA in the tumor microenvironment. Oncoimmunology, 4(6), e1008371. https://doi.org/10.1080/2162402x.2015.1008371

Sansone, P., Savini, C., Kurelac, I., Chang, Q., Amato, L. B., Strillacci, A., ... Bromberg, J. (2017). Packaging and transfer of mitochondrial DNA via exosomes regulate escape from dormancy in hormonal therapyresistant breast cancer. Proceedings of the National Academy of Sciences of the United States of America, 114(43), E9066-e9075. https://doi. org/10.1073/pnas.1704862114

Scherf, A., Lopez-Rubio, J. J., \& Riviere, L. (2008). Antigenic variation in Plasmodium falciparum. Annual Review of Microbiology, 62, 445-470. https://doi.org/10.1146/annurev.micro.61.080706.093134

Schorey, J. S., Cheng, Y., Singh, P. P., \& Smith, V. L. (2015). Exosomes and other extracellular vesicles in host-pathogen interactions. EMBO Reports, 16(1), 24-43. https://doi.org/10.15252/embr.201439363

Schorey, J. S., \& Harding, C. V. (2016). Extracellular vesicles and infectious diseases: New complexity to an old story. The Journal of Clinical Investigation, 126(4), 1181-1189. https://doi.org/10.1172/jci81132

Shenoy, G. N., Loyall, J., Maguire, O., Iyer, V., Kelleher, R. J. Jr., Minderman, H., ... Bankert, R. B. (2018). Exosomes associated with human ovarian tumors harbor a reversible checkpoint of T-cell responses. Cancer Immunology Research, 6(2), 236-247. https://doi.org/10.1158/23266066.cir-17-0113

Silverman, J. M., Clos, J., de'Oliveira, C. C., Shirvani, O., Fang, Y., Wang, C., ... Reiner, N. E. (2010). An exosome-based secretion pathway is responsible for protein export from Leishmania and communication with macrophages. Journal of Cell Science, 123(Pt 6), 842-852. https://doi. org/10.1242/jcs.056465

Silverman, J. M., \& Reiner, N. E. (2011). Leishmania exosomes deliver preemptive strikes to create an environment permissive for early infection. Frontiers in Cellular and Infection Microbiology, 1, 26-33. https://doi. org/10.3389/fcimb.2011.00026 
Simpson, R. J., Lim, J. W., Moritz, R. L., \& Mathivanan, S. (2009). Exosomes: Proteomic insights and diagnostic potential. Expert Review of Proteomics, 6(3), 267-283. https://doi.org/10.1586/epr.09.17

Sisquella, X., Ofir-Birin, Y., Pimentel, M. A., Cheng, L., Abou Karam, P., Sampaio, N. G., ... Regev-Rudzki, N. (2017). Malaria parasite DNA-harbouring vesicles activate cytosolic immune sensors. Nature Communications, 8(1), 1985. https://doi.org/10.1038/s41467-01702083-1

Szempruch, A. J., Dennison, L., Kieft, R., Harrington, J. M., \& Hajduk, S. L. (2016). Sending a message: Extracellular vesicles of pathogenic protozoan parasites. Nature Reviews. Microbiology, 14(11), 669-675. https://doi.org/10.1038/nrmicro.2016.110

Taylor, D. D., \& Gercel-Taylor, C. (2005). Tumour-derived exosomes and their role in cancer-associated T-cell signalling defects. British Journal of Cancer, 92(2), 305-311. https://doi.org/10.1038/sj.bjc.6602316

Taylor, D. D., Gercel-Taylor, C., Lyons, K. S., Stanson, J., \& Whiteside, T. L. (2003). T-cell apoptosis and suppression of T-cell receptor/CD3-zeta by Fas ligand-containing membrane vesicles shed from ovarian tumors. Clinical Cancer Research, 9(14), 5113-5119.

Thakur, B. K., Zhang, H., Becker, A., Matei, I., Huang, Y., Costa-Silva, B., ... Lyden, D. (2014). Double-stranded DNA in exosomes: A novel biomarker in cancer detection. Cell Research, 24(6), 766-769. https://doi. org/10.1038/cr.2014.44

Thery, C. (2011). Exosomes: Secreted vesicles and intercellular communications. F1000 Biology Reports, 3, 15-22. https://doi.org/10.3410/ b3-15

Torrecilhas, A. C., Schumacher, R. I., Alves, M. J., \& Colli, W. (2012). Vesicles as carriers of virulence factors in parasitic protozoan diseases. Microbes and Infection, 14(15), 1465-1474. https://doi.org/10.1016/j. micinf.2012.07.008
Verderio, C., Gabrielli, M., \& Giussani, P. (2018). Role of sphingolipids in the biogenesis and biological activity of extracellular vesicles. Journal of Lipid Research https://doi.org/10.1194/jlr.R083915, 59, 1325-1340.

Wisniewski, J. R., Zougman, A., Nagaraj, N., \& Mann, M. (2009). Universal sample preparation method for proteome analysis. Nature Methods, 6(5), 359-362. https://doi.org/10.1038/nmeth.1322

Yu, S., Liu, C., Su, K., Wang, J., Liu, Y., Zhang, L., ... Zhang, H. G. (2007). Tumor exosomes inhibit differentiation of bone marrow dendritic cells. Journal of Immunology, 178(11), 6867-6875. https://doi.org/10.4049/ jimmunol.178.11.6867

Zhang, H., Freitas, D., Kim, H. S., Fabijanic, K., Li, Z., Chen, H., ... Lyden, D. (2018). Identification of distinct nanoparticles and subsets of extracellular vesicles by asymmetric flow field-flow fractionation. Nature Cell Biology, 20(3), 332-343. https://doi.org/10.1038/s41556018-0040-4

\section{SUPPORTING INFORMATION}

Additional supporting information may be found online in the Supporting Information section at the end of the article.

How to cite this article: Demarta-Gatsi C, Rivkin A, Di Bartolo $\mathrm{V}$, et al. Histamine releasing factor and elongation factor 1 alpha secreted via malaria parasites extracellular vesicles promote immune evasion by inhibiting specific $\mathrm{T}$ cell responses. Cellular Microbiology. 2019;21:e13021. https://doi. org/10.1111/cmi.13021 